\title{
Salivary metabolomics reveals that metabolic alterations precede the onset of schizophrenia
}

\section{Authors}

Gaoping Cuil 1,\#, Ph.D.; Ying Qing ${ }^{1, \#, ~ P h . D . ; ~ M i n g h u i ~ L i ~}{ }^{1}$, M.S.; Liya Sun ${ }^{1}$, Ph.D.; Juan Zhang ${ }^{1}$, B.S.; Lei Feng², Ph.D.; Jing Li ${ }^{3}$, Ph.D.; Tianlu Chen ${ }^{4}$, Ph.D.; Jijun Wang ${ }^{5, *}$, M.D.; Chunling Wan ${ }^{1, *}$, Ph.D.

\# These authors contributed equally to this work.

${ }^{*}$ Corresponding authors: Jijun Wang, Chunling Wan.

\section{Author information}

1 Bio-X Institutes, Key Laboratory for the Genetics of Developmental and Neuropsychiatric Disorders, Ministry of Education, Shanghai Jiao Tong University, Shanghai 200030, China.

${ }^{2}$ Instrumental Analysis Center, Shanghai Jiao Tong University, Shanghai 200240, China.

${ }^{3}$ Department of Bioinformatics and Biostatistics, Shanghai Jiao Tong University, Shanghai 200240, China.

${ }^{4}$ Shanghai Key Laboratory of Diabetes Mellitus and Center for Translational Medicine, Shanghai Jiao Tong University Affiliated Sixth People's Hospital, Shanghai 200233, China.

${ }^{5}$ Shanghai Mental Health Centre, Shanghai Jiao Tong University School of Medicine, Shanghai Key Laboratory of Psychotic Disorders, Shanghai 200030, China. 


\section{Cover page}

\begin{tabular}{|l|l|}
\hline Name & Caption \\
\hline Figure S1 & $\begin{array}{l}\text { Score plot of PCA in QC samples and the samples of the three } \\
\text { groups, with QC samples clustered in the center. }\end{array}$ \\
\hline Table S1 & Differential metabolites among three groups. \\
\hline Table S2 & The diseases and biological functions in CHR subjects. \\
\hline Table S3 & The correlation network of salivary metabolites with symptoms of \\
& two disease statuses. \\
Table S4 & markers in the FES and HC groups, respectively. \\
\hline Table S5 & The correlations of salivary metabolites with salivary taxa. \\
\hline
\end{tabular}


Figure S1. Score plot of PCA in QC samples and the samples of the three groups, with QC samples clustered in the center.

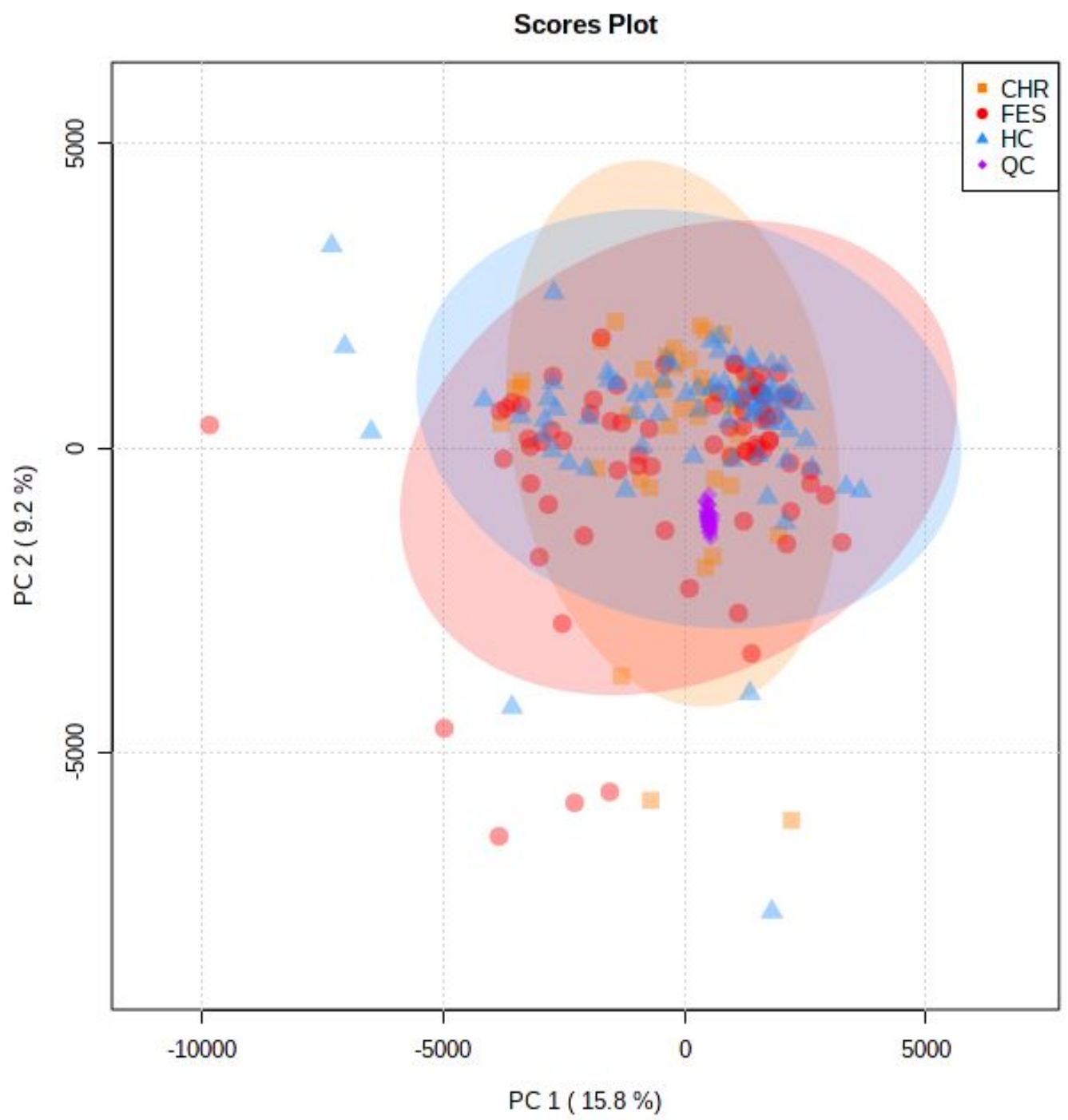


Table S1. Differential metabolites among three groups.

\begin{tabular}{|c|c|c|c|c|c|c|c|}
\hline Metabolites & VIP & q-value ${ }^{a}$ & FC & ROC & Classification & Pathway & Comparison \\
\hline Lysyl-Serine & 3.117 & 0.002 & 3.127 & 0.742 & Peptides & Amino acid metabolism & FES vs HC \\
\hline Flunisolide & 2.886 & 0.006 & 5.770 & 0.727 & Lipids & Lipid metabolism & FES vs HC \\
\hline Merodesmosine & 2.781 & 0.006 & 3.509 & 0.666 & Amino acids & Amino acid metabolism & FES vs HC \\
\hline $\begin{array}{l}\text { 2-hydroxy-3-(2,3,4-trimethoxyphenyl)propanoic } \\
\text { acid }\end{array}$ & 2.621 & 0.007 & -1.516 & 0.669 & Benzene derivatives & Benzene metabolism & FES vs HC \\
\hline Alanyl-Asparagine & 2.702 & 0.007 & 1.918 & 0.691 & Peptides & Amino acid metabolism & FES vs HC \\
\hline Alanyl-Serine & 2.687 & 0.007 & 3.481 & 0.691 & Peptides & Amino acid metabolism & FES vs HC \\
\hline Glycine & 2.650 & 0.007 & 1.475 & 0.645 & Amino acids & Purine metabolism & FES vs HC \\
\hline L-2-hydroxyglutaric acid & 2.472 & 0.010 & 2.491 & 0.712 & Organic acids & Lysine degradation & FES vs HC \\
\hline Itaconic acid & 2.463 & 0.010 & 2.605 & 0.709 & Fatty acids & $\begin{array}{l}\text { C5-Branched dibasic acid } \\
\text { metabolism }\end{array}$ & FES vs HC \\
\hline (S,S)-Nt-Histidinylalanine & 2.220 & 0.010 & 2.735 & 0.660 & Amino acids & Amino acid metabolism & FES vs HC \\
\hline APGPR Enterostatin & 2.297 & 0.010 & 1.587 & 0.625 & Peptides & Amino acid metabolism & FES vs HC \\
\hline Metanephrine & 2.560 & 0.010 & -1.529 & 0.677 & Benzene derivatives & Tyrosine metabolism & FES vs HC \\
\hline Glycyl-Lysine & 2.291 & 0.010 & 1.474 & 0.659 & Peptides & Amino acid metabolism & FES vs HC \\
\hline Pelargonic acid & 2.129 & 0.010 & 1.384 & 0.642 & Fatty acids & Fatty acid metabolism & FES vs HC \\
\hline Flumethasone Pivalate & 2.395 & 0.010 & 1.798 & 0.617 & Lipids & Lipid metabolism & FES vs HC \\
\hline 5a-Tetrahydrocortisol & 2.441 & 0.010 & -1.371 & 0.612 & Lipids & Lipid metabolism & FES vs HC \\
\hline S-carboxymethyl-L-cysteine & 2.415 & 0.010 & -1.397 & 0.590 & Amino acids & Amino acid metabolism & FES vs HC \\
\hline Glycyltyrosine & 2.172 & 0.010 & 2.868 & 0.652 & Peptides & Amino acid metabolism & FES vs HC \\
\hline Uric acid & 2.244 & 0.011 & 1.478 & 0.665 & Nucleotides & Purine metabolism & FES vs HC \\
\hline Urate radical & 2.260 & 0.011 & 1.399 & 0.646 & Nucleotides & Nucleotide metabolism & FES vs HC \\
\hline Hydroquinone & 1.924 & 0.011 & 1.477 & 0.595 & Benzene derivatives & Tyrosine metabolism & FES vs HC \\
\hline Fumarycarnitine & 2.485 & 0.012 & -1.692 & 0.720 & Lipids & Lipid metabolism & FES vs HC \\
\hline
\end{tabular}




\begin{tabular}{|c|c|c|c|c|c|c|c|}
\hline (S)-3-Sulfonatolactate & 2.368 & 0.012 & -1.724 & 0.656 & Organic acids & $\begin{array}{l}\text { Cysteine and methionine } \\
\text { metabolism }\end{array}$ & FES vs HC \\
\hline Norepinephrine sulfate & 2.257 & 0.012 & -1.451 & 0.631 & Benzene derivatives & Tyrosine metabolism & FES vs HC \\
\hline Toremifene & 2.310 & 0.012 & 2.473 & 0.652 & Others & Others & FES vs HC \\
\hline dUMP & 2.360 & 0.012 & -1.287 & 0.585 & Nucleotides & Pyrimidine metabolism & FES vs HC \\
\hline (S)-5-Diphosphomevalonic acid & 2.227 & 0.012 & -1.195 & 0.624 & Organic acids & $\begin{array}{l}\text { Biosynthesis of terpenoids } \\
\text { and steroids }\end{array}$ & FES vs HC \\
\hline 2(R)-hydroxydocosanoic acid & 2.242 & 0.012 & -1.743 & 0.652 & Fatty acids & Fatty acid metabolism & FES vs HC \\
\hline Cerebronic acid & 2.209 & 0.013 & -1.476 & 0.656 & Fatty acids & Fatty acid metabolism & FES vs HC \\
\hline N2-Succinoylarginine & 2.110 & 0.013 & 2.501 & 0.703 & Amino acids & Amino acid metabolism & FES vs HC \\
\hline Squamosinin A & 2.291 & 0.013 & -1.640 & 0.591 & Lipids & Lipid metabolism & FES vs $\mathrm{HC}$ \\
\hline B-Sulfinyl pyruvate & 2.269 & 0.013 & -1.665 & 0.658 & Organic acids & $\begin{array}{l}\text { Cysteine and methionine } \\
\text { metabolism }\end{array}$ & FES vs HC \\
\hline Saccharin & 2.302 & 0.013 & -1.141 & 0.568 & Benzene derivatives & Tsate transduction & FES vs HC \\
\hline Orotidine & 2.250 & 0.013 & -1.239 & 0.635 & Nucleotides & Pyrimidine metabolism & FES vs HC \\
\hline Sodium Tetradecyl Sulfate & 2.091 & 0.014 & -1.397 & 0.613 & Others & Others & FES vs HC \\
\hline Normetanephrine & 2.316 & 0.015 & -1.257 & 0.644 & Benzene derivatives & Tyrosine metabolism & FES vs HC \\
\hline 5-Hydroxyindoleacetaldehyde & 2.190 & 0.015 & -2.284 & 0.689 & Indoles & Tryptophan metabolism & FES vs HC \\
\hline ETHYL 3-INDOLEACETATE & 1.941 & 0.015 & -1.134 & 0.611 & Others & Others & FES vs HC \\
\hline Carnosine & 2.046 & 0.017 & 1.295 & 0.618 & Peptides & Histidine metabolism & FES vs HC \\
\hline Lactic acid & 1.923 & 0.019 & -1.325 & 0.651 & Organic acids & $\begin{array}{l}\text { Glycolysis / } \\
\text { Gluconeogenesis }\end{array}$ & FES vs HC \\
\hline 1-Methyladenosine & 1.888 & 0.019 & 1.555 & 0.651 & Nucleotides & Nucleotide metabolism & FES vs $\mathrm{HC}$ \\
\hline Histidinyl-Phenylalanine & 1.785 & 0.020 & 2.869 & 0.675 & Peptides & Amino acid metabolism & FES vs HC \\
\hline Dopamine 3-O-sulfate & 2.096 & 0.020 & -1.453 & 0.639 & Benzene derivatives & Tyrosine metabolism & FES vs $\mathrm{HC}$ \\
\hline
\end{tabular}




\begin{tabular}{|c|c|c|c|c|c|c|c|}
\hline 3-Hydroxymethylglutaric acid & 2.106 & 0.020 & -1.447 & 0.616 & Organic acids & $\begin{array}{l}\text { Valine, leucine and } \\
\text { isoleucine degradation }\end{array}$ & FES vs HC \\
\hline $\mathrm{C} 23: 0$ & 1.979 & 0.020 & -1.143 & 0.570 & Fatty acids & Fatty acid metabolism & FES vs HC \\
\hline Histidinyl-Histidine & 1.621 & 0.020 & 1.076 & 0.555 & Peptides & Amino acid metabolism & FES vs HC \\
\hline D-Glycero-D-galacto-heptitol & 1.946 & 0.020 & 2.118 & 0.628 & Carbohydrates & Carbohydrate metabolism & FES vs HC \\
\hline Acetoacetic acid & 1.901 & 0.020 & 1.592 & 0.665 & Organic acids & $\begin{array}{l}\text { Synthesis and degradation of } \\
\text { ketone bodies }\end{array}$ & FES vs HC \\
\hline L-Dopa & 1.907 & 0.020 & -1.064 & 0.563 & Amino acids & Tyrosine metabolism & FES vs HC \\
\hline 5-hydroxyindoleacetic acid & 2.105 & 0.023 & -1.645 & 0.707 & Indoles & Tryptophan metabolism & FES vs HC \\
\hline L-Cyclo(alanylglycyl) & 1.842 & 0.024 & 2.464 & 0.673 & Others & Others & FES vs HC \\
\hline Glutaminyltyrosine & 1.852 & 0.024 & 2.372 & 0.648 & Peptides & Amino acid metabolism & FES vs HC \\
\hline $\begin{array}{l}\text { a-L-threo-4-Hex-4-enopyranuronosyl-D- } \\
\text { galacturonic acid }\end{array}$ & 1.761 & 0.024 & -1.347 & 0.610 & Carbohydrates & Carbohydrate metabolism & FES vs $\mathrm{HC}$ \\
\hline Glutamylhistidine & 1.712 & 0.024 & 1.680 & 0.619 & Peptides & Amino acid metabolism & FES vs HC \\
\hline Caryoptosidic acid & 1.771 & 0.024 & 2.015 & 0.605 & Lipids & Lipid metabolism & FES vs HC \\
\hline Serylglutamine & 1.951 & 0.024 & 3.149 & 0.730 & Peptides & Amino acid metabolism & FES vs HC \\
\hline Nopalinic acid & 1.754 & 0.024 & -1.268 & 0.649 & Amino acids & Amino acid metabolism & FES vs HC \\
\hline Valyl-Alanine & 1.592 & 0.025 & -2.114 & 0.629 & Peptides & Amino acid metabolism & FES vs HC \\
\hline riboflavin & 1.883 & 0.025 & -1.811 & 0.672 & Others & Riboflavin metabolism & FES vs HC \\
\hline $\begin{array}{l}\text { 5-hydroxy-4-(4-hydroxyphenyl)-8,8-dimethyl- } \\
\text { 2H,8H-pyrano[2,3-f]chromen-2-one }\end{array}$ & 1.868 & 0.027 & -1.718 & 0.688 & Others & Others & FES vs HC \\
\hline Alanyl-Glutamine & 1.951 & 0.027 & 2.024 & 0.633 & Peptides & Amino acid metabolism & FES vs HC \\
\hline Isocitric acid & 1.637 & 0.027 & 1.181 & 0.564 & Organic acids & Citrate cycle (TCA cycle) & FES vs HC \\
\hline L-beta-aspartyl-L-aspartic acid & 1.989 & 0.029 & -1.450 & 0.669 & Amino acids & Amino acid metabolism & FES vs HC \\
\hline Furoic acid & 1.579 & 0.030 & 1.050 & 0.528 & Others & Furfural degradation & FES vs HC \\
\hline 2-Hydroxypyridine & 1.833 & 0.031 & -1.291 & 0.597 & Nucleotides & Nucleotide metabolism & FES vs HC \\
\hline
\end{tabular}




\begin{tabular}{|c|c|c|c|c|c|c|c|}
\hline L-beta-aspartyl-L-alanine & 1.655 & 0.032 & -1.842 & 0.654 & Peptides & Amino acid metabolism & FES vs HC \\
\hline Prostaglandin A1 & 1.395 & 0.033 & -2.430 & 0.625 & Fatty acids & Fatty acid metabolism & FES vs HC \\
\hline Prolyl-Glutamine & 1.876 & 0.033 & 2.280 & 0.643 & Peptides & Amino acid metabolism & FES vs HC \\
\hline 5'-Carboxy-gamma-chromanol & 1.518 & 0.033 & -1.564 & 0.583 & Others & Others & FES vs HC \\
\hline 1-Naphthylamine & 1.599 & 0.033 & -1.096 & 0.519 & Others & $\begin{array}{l}\text { Metabolism of xenobiotics } \\
\text { by cytochrome } \mathrm{P} 450\end{array}$ & FES vs HC \\
\hline Methohexital & 1.864 & 0.034 & 1.564 & 0.608 & Others & Others & FES vs HC \\
\hline Val-Val-Val & 1.856 & 0.035 & 2.720 & 0.681 & Peptides & Amino acid metabolism & FES vs HC \\
\hline PC(P-16:0/18:3(6Z,9Z,12Z)) & 1.739 & 0.035 & -1.107 & 0.583 & Lipids & Lipid metabolism & FES vs HC \\
\hline Isopentenyl pyrophosphate & 1.621 & 0.036 & 1.321 & 0.604 & Lipids & $\begin{array}{l}\text { Terpenoid backbone } \\
\text { biosynthesis }\end{array}$ & FES vs HC \\
\hline 2-Hydroxybenzaldehyde & 1.793 & 0.038 & 1.910 & 0.683 & Benzene derivatives & $\begin{array}{l}\text { Degradation of aromatic } \\
\text { compounds }\end{array}$ & FES vs HC \\
\hline 2-Aminophenol & 1.586 & 0.038 & -1.332 & 0.594 & Benzene derivatives & Tryptophan metabolism & FES vs HC \\
\hline Succinic acid & 1.566 & 0.040 & 1.475 & 0.624 & Organic acids & Citrate cycle (TCA cycle) & FES vs HC \\
\hline phenylacetylglycine & 1.924 & 0.040 & -1.488 & 0.650 & Amino acids & Phenylalanine metabolism & FES vs HC \\
\hline 24,25-Dihydroxyvitamin D & 1.749 & 0.040 & -1.080 & 0.580 & Others & Others & FES vs HC \\
\hline Serylserine & 1.468 & 0.041 & 2.337 & 0.602 & Peptides & Amino acid metabolism & FES vs HC \\
\hline p-Octopamine & 1.808 & 0.041 & -1.260 & 0.632 & Others & $\begin{array}{l}\text { Neuroactive ligand-receptor } \\
\text { interaction }\end{array}$ & FES vs HC \\
\hline Tyrosyl-Isoleucine & 1.502 & 0.042 & 2.235 & 0.588 & Peptides & Amino acid metabolism & FES vs HC \\
\hline N-Caffeoyltryptophan & 1.612 & 0.042 & -1.400 & 0.609 & Amino acids & Amino acid metabolism & FES vs HC \\
\hline Histidinyl-Lysine & 1.347 & 0.042 & -1.043 & 0.548 & Others & Others & FES vs HC \\
\hline Muricatacin & 1.566 & 0.043 & 1.340 & 0.571 & Others & Others & FES vs HC \\
\hline gamma-Glutamylisoleucine & 1.405 & 0.043 & -3.116 & 0.600 & Peptides & Amino acid metabolism & FES vs HC \\
\hline 2-(1-Ethoxyethoxy)propanoic acid & 1.327 & 0.043 & -1.118 & 0.539 & Organic acids & Organic acid metabolism & FES vs HC \\
\hline
\end{tabular}




\begin{tabular}{|c|c|c|c|c|c|c|c|}
\hline Hydroxyprolyl-Valine & 1.589 & 0.044 & -1.785 & 0.647 & Peptides & Amino acid metabolism & FES vs HC \\
\hline $\begin{array}{l}\text { (3beta,17alpha,23S,24S)-17,23-Epoxy-3,24,29- } \\
\text { trihydroxy-27-norlanost-8-en-15-one }\end{array}$ & 1.538 & 0.045 & -1.483 & 0.624 & Lipids & Lipid metabolism & FES vs HC \\
\hline Cer(d18:1/14:0) & 1.693 & 0.045 & -1.196 & 0.571 & Lipids & Lipid metabolism & FES vs HC \\
\hline Glutamine & 1.675 & 0.045 & 5.296 & 0.691 & Amino acids & Pyrimidine metabolism & FES vs HC \\
\hline Asparaginyl-Tyrosine & 1.387 & 0.045 & 1.535 & 0.574 & Peptides & Amino acid metabolism & FES vs HC \\
\hline Vanillylmandelic acid & 1.429 & 0.045 & -1.106 & 0.552 & Benzene derivatives & Tyrosine metabolism & FES vs HC \\
\hline 5-Sulfoxymethylfurfural & 1.220 & 0.045 & 1.039 & 0.529 & Others & Others & FES vs HC \\
\hline Glutamylalanine & 1.729 & 0.045 & -2.298 & 0.648 & Peptides & Amino acid metabolism & FES vs $\mathrm{HC}$ \\
\hline Tetrahydrobiopterin & 1.235 & 0.045 & -1.212 & 0.546 & Others & Folate biosynthesis & FES vs HC \\
\hline Alpha-Hydroxyisobutyric acid & 1.471 & 0.046 & 1.302 & 0.635 & Organic acids & Organic acid metabolism & FES vs $\mathrm{HC}$ \\
\hline Trihydroxycoprostanoic acid & 1.689 & 0.047 & -1.232 & 0.591 & Lipids & Lipid metabolism & FES vs HC \\
\hline 2-methyl-3-(sulfooxy)propanoic acid & 1.608 & 0.047 & -1.079 & 0.546 & Organic acids & Organic acid metabolism & FES vs HC \\
\hline 1-Methylhistidine & 1.423 & 0.048 & 1.007 & 0.527 & Amino acids & Amino acid metabolism & FES vs HC \\
\hline $\begin{array}{l}\text { Glucosyl (2E,6E,10x)-10,11-dihydroxy-2,6- } \\
\text { farnesadienoate }\end{array}$ & 1.403 & 0.050 & 1.472 & 0.559 & Lipids & Lipid metabolism & FES vs HC \\
\hline O-Acetyl-L-homoserine & 1.393 & 0.050 & 1.604 & 0.574 & Amino acids & $\begin{array}{l}\text { Cysteine and methionine } \\
\text { metabolism }\end{array}$ & FES vs HC \\
\hline $\begin{array}{l}\text { 2-hydroxy-3-(2,3,4-trimethoxyphenyl)propanoic } \\
\text { acid }\end{array}$ & 2.658 & 0.000 & -1.954 & 0.743 & Benzene derivatives & Benzene metabolism & CHR vs HC \\
\hline $\begin{array}{l}\text { (3beta,17alpha,23S,24S)-17,23-Epoxy-3,24,29- } \\
\text { trihydroxy-27-norlanost-8-en-15-one }\end{array}$ & 2.842 & 0.000 & -1.882 & 0.742 & Lipids & Lipid metabolism & CHR vs HC \\
\hline 4-OH-Retinal & 2.403 & 0.000 & -2.543 & 0.740 & Lipids & Lipid metabolism & CHR vs HC \\
\hline Lysyl-Serine & 2.651 & 0.001 & 2.101 & 0.649 & Peptides & Amino acid metabolism & CHR vs HC \\
\hline C20:5(cis-5,8,11,14,17) & 1.948 & 0.002 & -2.519 & 0.704 & Fatty acids & $\begin{array}{l}\text { Biosynthesis of unsaturated } \\
\text { fatty acids }\end{array}$ & CHR vs HC \\
\hline
\end{tabular}




\begin{tabular}{|c|c|c|c|c|c|c|c|}
\hline Phaseolic acid & 3.041 & 0.002 & 5.762 & 0.751 & Organic acids & Organic acid metabolism & CHR vs HC \\
\hline Aniline-2-sulfonic acid & 2.934 & 0.002 & -1.986 & 0.749 & Others & Others & $\mathrm{CHR}$ vs $\mathrm{HC}$ \\
\hline C14:1(cis-9) & 1.436 & 0.002 & -1.533 & 0.607 & Fatty acids & Fatty acid metabolism & CHR vs HC \\
\hline Alanyl-Asparagine & 2.483 & 0.005 & 2.577 & 0.680 & Peptides & Amino acid metabolism & CHR vs HC \\
\hline N2-Succinoylarginine & 2.035 & 0.005 & 2.456 & 0.635 & Amino acids & Amino acid metabolism & CHR vs HC \\
\hline riboflavin & 1.969 & 0.005 & -1.720 & 0.692 & Others & Riboflavin metabolism & CHR vs HC \\
\hline $\operatorname{Cer}(\mathrm{d} 18: 1 / 14: 0)$ & 2.313 & 0.005 & -1.857 & 0.714 & Lipids & Lipid metabolism & CHR vs HC \\
\hline cis-5-Tetradecenoylcarnitine & 2.073 & 0.005 & -1.805 & 0.770 & Lipids & Lipid metabolism & CHR vs HC \\
\hline C18:1(cis-9) & 1.849 & 0.005 & -1.508 & 0.624 & Fatty acids & Fatty acid biosynthesis & $\mathrm{CHR}$ vs $\mathrm{HC}$ \\
\hline C16:1(cis-9) & 1.674 & 0.005 & -1.404 & 0.581 & Fatty acids & Fatty acid biosynthesis & CHR vs HC \\
\hline PG(16:1(9Z)/18:1(11Z)) & 2.038 & 0.006 & -1.648 & 0.660 & Lipids & Lipid metabolism & CHR vs HC \\
\hline cis-2-Methylaconitate & 2.062 & 0.006 & -1.679 & 0.675 & Organic acids & Propanoate metabolism & CHR vs HC \\
\hline Asparaginyl-Glycine & 2.069 & 0.007 & 1.697 & 0.628 & Peptides & Amino acid metabolism & CHR vs HC \\
\hline 2-Hydroxypyridine & 2.011 & 0.009 & -1.906 & 0.633 & Nucleotides & Nucleotide metabolism & CHR vs HC \\
\hline PG(18:2(9Z,12Z)/16:0) & 1.993 & 0.009 & -1.993 & 0.719 & Lipids & Lipid metabolism & CHR vs HC \\
\hline $\begin{array}{l}\text { Methyl 3-(2,3-dihydroxy-3-methylbutyl)-4- } \\
\text { hydroxybenzoate }\end{array}$ & 1.731 & 0.009 & -1.384 & 0.670 & Benzene derivatives & Benzene metabolism & CHR vs HC \\
\hline Acetoin & 2.443 & 0.009 & -1.748 & 0.665 & Others & Others & $\mathrm{CHR}$ vs $\mathrm{HC}$ \\
\hline $\begin{array}{l}\text { 6-hydroxy-7-methoxy-2H-1,3-benzodioxole-5- } \\
\text { carboxylic acid }\end{array}$ & 1.579 & 0.009 & -1.357 & 0.581 & Benzene derivatives & Benzene metabolism & CHR vs HC \\
\hline Metronidazole & 1.871 & 0.010 & 1.653 & 0.590 & Others & Others & CHR vs HC \\
\hline 15-Deoxy-d-12,14-PGJ2 & 1.089 & 0.010 & -1.008 & 0.549 & Lipids & $\begin{array}{l}\text { Arachidonic acid } \\
\text { metabolism }\end{array}$ & CHR vs HC \\
\hline PG(16:1(9Z)/18:0) & 1.712 & 0.011 & -1.707 & 0.672 & Lipids & Lipid metabolism & CHR vs HC \\
\hline 11b-Hydroxyprogesterone & 1.433 & 0.011 & -1.408 & 0.642 & Lipids & $\begin{array}{l}\text { Steroid hormone } \\
\text { biosynthesis }\end{array}$ & CHR vs HC \\
\hline
\end{tabular}




\begin{tabular}{|c|c|c|c|c|c|c|c|}
\hline Nicotinic acid adenine dinucleotide & 1.558 & 0.011 & -1.312 & 0.572 & Nucleotides & $\begin{array}{l}\text { Nicotinate and nicotinamide } \\
\text { metabolism }\end{array}$ & CHR vs HC \\
\hline Glycine & 2.112 & 0.012 & 1.418 & 0.614 & Amino acids & Purine metabolism & CHR vs HC \\
\hline (S)-3-Sulfonatolactate & 1.675 & 0.012 & -2.119 & 0.664 & Organic acids & $\begin{array}{l}\text { Cysteine and methionine } \\
\text { metabolism }\end{array}$ & CHR vs HC \\
\hline Epicatechin & 1.897 & 0.012 & -1.423 & 0.666 & Others & Flavonoid biosynthesis & CHR vs HC \\
\hline PE-NMe2(14:1(9Z)/16:0) & 1.716 & 0.012 & -1.506 & 0.605 & Lipids & Lipid metabolism & CHR vs HC \\
\hline $\operatorname{PE}(16: 0 / 16: 1(9 Z))$ & 1.718 & 0.012 & -1.200 & 0.591 & Lipids & Lipid metabolism & CHR vs HC \\
\hline Ethyl beta-D-glucopyranoside & 1.356 & 0.012 & -1.360 & 0.548 & Carbohydrates & Carbohydrate metabolism & CHR vs HC \\
\hline Dopamine 3-O-sulfate & 1.718 & 0.013 & -1.358 & 0.637 & Benzene derivatives & Tyrosine metabolism & CHR vs HC \\
\hline Ceanothic acid & 1.946 & 0.013 & -1.921 & 0.707 & Lipids & Lipid metabolism & CHR vs HC \\
\hline Hydroquinone & 1.897 & 0.014 & 1.900 & 0.610 & Benzene derivatives & Tyrosine metabolism & CHR vs HC \\
\hline 2-Hydroxybenzaldehyde & 1.496 & 0.014 & 1.939 & 0.654 & Benzene derivatives & $\begin{array}{l}\text { Degradation of aromatic } \\
\text { compounds }\end{array}$ & CHR vs HC \\
\hline 2-Aminophenol & 2.215 & 0.014 & -2.000 & 0.637 & Benzene derivatives & Tryptophan metabolism & CHR vs HC \\
\hline Tridecanoic acid & 1.243 & 0.014 & -1.171 & 0.541 & Fatty acids & Fatty acid metabolism & CHR vs HC \\
\hline Homogentisic acid & 1.800 & 0.014 & -1.781 & 0.584 & Organic acids & Tyrosine metabolism & CHR vs HC \\
\hline 2,3-Butanediol glucoside & 1.279 & 0.014 & -1.276 & 0.512 & Carbohydrates & Carbohydrate metabolism & CHR vs HC \\
\hline B-Sulfinyl pyruvate & 1.590 & 0.014 & -2.017 & 0.664 & Organic acids & $\begin{array}{l}\text { Cysteine and methionine } \\
\text { metabolism }\end{array}$ & CHR vs HC \\
\hline $\mathrm{C} 24: 0$ & 1.373 & 0.014 & -1.398 & 0.584 & Fatty acids & $\begin{array}{l}\text { Biosynthesis of unsaturated } \\
\text { fatty acids }\end{array}$ & CHR vs HC \\
\hline Prenyl glucoside & 2.723 & 0.014 & 1.917 & 0.733 & Lipids & Lipid metabolism & CHR vs HC \\
\hline 4-cresyl sulfate & 1.692 & 0.014 & 2.455 & 0.592 & Benzene derivatives & Tyrosine metabolism & CHR vs HC \\
\hline$(9 \mathrm{~S}, 10 \mathrm{~S})-9,10$-dihydroxyoctadecanoate & 1.346 & 0.016 & 2.533 & 0.647 & Fatty acids & Fatty acid metabolism & CHR vs HC \\
\hline Phytocassane C & 1.199 & 0.016 & -1.130 & 0.602 & Lipids & Lipid metabolism & CHR vs HC \\
\hline
\end{tabular}




\begin{tabular}{|c|c|c|c|c|c|c|c|}
\hline Norepinephrine sulfate & 1.716 & 0.017 & -1.764 & 0.672 & Benzene derivatives & Tyrosine metabolism & CHR vs HC \\
\hline dUMP & 1.342 & 0.017 & -1.321 & 0.587 & Nucleotides & Pyrimidine metabolism & $\mathrm{CHR}$ vs $\mathrm{HC}$ \\
\hline L-2-hydroxyglutaric acid & 2.257 & 0.017 & 3.035 & 0.717 & Organic acids & Lysine degradation & CHR vs HC \\
\hline Itaconic acid & 2.248 & 0.017 & 3.343 & 0.716 & Fatty acids & $\begin{array}{l}\text { C5-Branched dibasic acid } \\
\text { metabolism }\end{array}$ & $\mathrm{CHR}$ vs $\mathrm{HC}$ \\
\hline 3-Hydroxymethylglutaric acid & 1.377 & 0.017 & -1.658 & 0.565 & Organic acids & $\begin{array}{l}\text { Valine, leucine and } \\
\text { isoleucine degradation }\end{array}$ & $\mathrm{CHR}$ vs $\mathrm{HC}$ \\
\hline Genipinic acid & 1.400 & 0.017 & -1.276 & 0.610 & Organic acids & Organic acid metabolism & CHR vs HC \\
\hline $\begin{array}{l}\text { 3-[4-methoxy-3-(sulfooxy)phenyl]propanoic } \\
\text { acid }\end{array}$ & 2.338 & 0.017 & -3.670 & 0.690 & Benzene derivatives & Benzene metabolism & $\mathrm{CHR}$ vs $\mathrm{HC}$ \\
\hline $\begin{array}{l}\text { [4-(2-phenylcyclobutyl)phenyl]oxidanesulfonic } \\
\text { acid }\end{array}$ & 1.748 & 0.017 & -1.639 & 0.617 & Others & Others & CHR vs HC \\
\hline 5'-Carboxy-gamma-chromanol & 2.532 & 0.018 & -3.198 & 0.652 & Others & Others & CHR vs HC \\
\hline Methohexital & 1.472 & 0.018 & 1.634 & 0.611 & Others & Others & CHR vs HC \\
\hline Aprobarbital & 1.876 & 0.018 & -1.120 & 0.634 & Others & Others & CHR vs HC \\
\hline PG(16:1(9Z)/16:0) & 1.715 & 0.018 & -1.275 & 0.610 & Lipids & Lipid metabolism & $\mathrm{CHR}$ vs $\mathrm{HC}$ \\
\hline $\operatorname{PE}(16: 0 / 18: 2(9 Z, 12 Z))$ & 1.563 & 0.019 & -1.441 & 0.576 & Lipids & Lipid metabolism & CHR vs HC \\
\hline Glycyltyrosine & 1.282 & 0.019 & 2.481 & 0.628 & Peptides & Amino acid metabolism & CHR vs HC \\
\hline C17:1(cis-10) & 1.577 & 0.020 & -1.461 & 0.634 & Fatty acids & Fatty acid metabolism & CHR vs HC \\
\hline Lipoamide & 1.148 & 0.020 & 1.120 & 0.557 & Others & Others & CHR vs HC \\
\hline N-acetyl-L-2-aminoadipate(2-) & 1.021 & 0.021 & -1.810 & 0.558 & Amino acids & Amino acid metabolism & CHR vs HC \\
\hline $\begin{array}{l}\text { Glucosyl (2E,6E,10x)-10,11-dihydroxy-2,6- } \\
\text { farnesadienoate }\end{array}$ & 1.833 & 0.022 & 2.990 & 0.664 & Lipids & Lipid metabolism & CHR vs HC \\
\hline 11-Dehydro-thromboxane B2 & 1.793 & 0.022 & -1.208 & 0.631 & Lipids & $\begin{array}{l}\text { Arachidonic acid } \\
\text { metabolism }\end{array}$ & CHR vs HC \\
\hline Urate radical & 2.092 & 0.022 & 1.272 & 0.653 & Nucleotides & Nucleotide metabolism & CHR vs HC \\
\hline
\end{tabular}




\begin{tabular}{|c|c|c|c|c|c|c|c|}
\hline p-Aminobenzoic acid & 1.756 & 0.022 & -1.205 & 0.636 & Benzene derivatives & Aminobenzoate degradation & CHR vs HC \\
\hline Glucosamine 6-sulfate & 1.290 & 0.022 & -1.124 & 0.547 & Carbohydrates & Carbohydrate metabolism & CHR vs HC \\
\hline Glucosylisomaltol & 1.276 & 0.022 & -1.331 & 0.597 & Carbohydrates & Carbohydrate metabolism & CHR vs HC \\
\hline 7-Methylguanine & 1.140 & 0.022 & -1.240 & 0.549 & Nucleotides & Nucleotide metabolism & CHR vs HC \\
\hline Glycyl-Lysine & 1.749 & 0.022 & 1.124 & 0.581 & Peptides & Amino acid metabolism & CHR vs HC \\
\hline L-beta-aspartyl-L-aspartic acid & 1.378 & 0.022 & -1.776 & 0.694 & Amino acids & Amino acid metabolism & CHR vs HC \\
\hline 9E,11E-Octadecadienoic acid & 1.421 & 0.022 & -1.436 & 0.600 & Fatty acids & Fatty acid metabolism & CHR vs HC \\
\hline Junicedral & 1.912 & 0.023 & -1.661 & 0.683 & Lipids & Lipid metabolism & CHR vs HC \\
\hline Metanephrine & 1.263 & 0.023 & -1.303 & 0.599 & Benzene derivatives & Tyrosine metabolism & CHR vs HC \\
\hline Linalyl caprylate & 1.998 & 0.023 & -1.686 & 0.670 & Lipids & Lipid metabolism & CHR vs HC \\
\hline Citrulline & 1.452 & 0.023 & 1.607 & 0.676 & Amino acids & Arginine biosynthesis & CHR vs HC \\
\hline Campesterol & 1.235 & 0.023 & -1.610 & 0.673 & Lipids & Steroid biosynthesis & CHR vs HC \\
\hline Tiglylcarnitine & 1.858 & 0.023 & -1.364 & 0.655 & Lipids & Lipid metabolism & CHR vs HC \\
\hline Thiodiacetic acid & 1.083 & 0.023 & 1.248 & 0.611 & Organic acids & $\begin{array}{l}\text { Metabolism of xenobiotics } \\
\text { by cytochrome P450 }\end{array}$ & CHR vs HC \\
\hline Indole-3-methyl acetate & 1.913 & 0.024 & -1.120 & 0.639 & Indoles & Indole metabolism & CHR vs HC \\
\hline 3-(4,5-dihydroxy-2,3-dimethoxyphenyl)propanal & 1.320 & 0.024 & -1.556 & 0.600 & Benzene derivatives & Benzene metabolism & CHR vs HC \\
\hline 3-Methylxanthine & 1.279 & 0.024 & -1.188 & 0.568 & Nucleotides & Caffeine metabolism & CHR vs HC \\
\hline $\operatorname{PE}(14: 0 / 16: 0)$ & 1.045 & 0.024 & -1.299 & 0.550 & Lipids & Lipid metabolism & CHR vs HC \\
\hline 5-L-Glutamyl-taurine & 2.017 & 0.024 & 1.452 & 0.670 & Amino acids & $\begin{array}{l}\text { Taurine and hypotaurine } \\
\text { metabolism }\end{array}$ & CHR vs HC \\
\hline Glutamylalanine & 1.079 & 0.024 & -1.167 & 0.557 & Peptides & Amino acid metabolism & CHR vs HC \\
\hline Pterin & 1.172 & 0.024 & -1.714 & 0.645 & Others & Others & CHR vs HC \\
\hline Myricetin & 1.843 & 0.024 & -1.621 & 0.675 & Benzene derivatives & Flavonoid biosynthesis & CHR vs HC \\
\hline 5-hydroxyindole & 1.581 & 0.024 & -1.281 & 0.648 & Indoles & Indole metabolism & CHR vs HC \\
\hline Pyridoxic acid & 1.126 & 0.025 & -1.244 & 0.557 & Others & Vitamin B6 metabolism & CHR vs HC \\
\hline
\end{tabular}




\begin{tabular}{|c|c|c|c|c|c|c|c|}
\hline 3,4-Dihydroxybenzylamine & 1.099 & 0.027 & -1.739 & 0.648 & Benzene derivatives & Benzene metabolism & CHR vs HC \\
\hline Uric acid & 2.054 & 0.027 & 1.356 & 0.682 & Nucleotides & Purine metabolism & CHR vs HC \\
\hline Toremifene & 1.407 & 0.027 & 2.762 & 0.620 & Others & Others & CHR vs HC \\
\hline 5-Hydroxyindoleacetaldehyde & 1.196 & 0.027 & -1.838 & 0.630 & Indoles & Tryptophan metabolism & CHR vs HC \\
\hline Prolyl-Glutamine & 1.386 & 0.027 & 2.538 & 0.611 & Peptides & Amino acid metabolism & CHR vs HC \\
\hline Prostaglandin D2 & 2.715 & 0.027 & -2.160 & 0.763 & Lipids & $\begin{array}{l}\text { Arachidonic acid } \\
\text { metabolism }\end{array}$ & CHR vs HC \\
\hline Deoxyguanosine & 1.678 & 0.027 & -4.144 & 0.696 & Nucleotides & Purine metabolism & CHR vs HC \\
\hline 3,4-Methylenesebacic acid & 1.985 & 0.027 & 1.728 & 0.671 & Fatty acids & Fatty acid metabolism & CHR vs HC \\
\hline Cortisol & 1.685 & 0.027 & -1.392 & 0.636 & Lipids & $\begin{array}{l}\text { Steroid hormone } \\
\text { biosynthesis }\end{array}$ & CHR vs HC \\
\hline 9Z-Eicosenoic acid & 1.420 & 0.027 & -1.283 & 0.601 & Fatty acids & Fatty acid metabolism & CHR vs HC \\
\hline [(oxolan-2-yl)methoxy]sulfonic acid & 1.623 & 0.027 & -1.546 & 0.600 & Others & Others & CHR vs HC \\
\hline L-Cyclo(alanylglycyl) & 1.941 & 0.028 & 2.990 & 0.617 & Others & Others & CHR vs HC \\
\hline Phenylalanyl-Arginine & 1.553 & 0.028 & -1.612 & 0.648 & Peptides & Amino acid metabolism & CHR vs HC \\
\hline Monoethyl malonic acid & 1.057 & 0.028 & -1.406 & 0.531 & Organic acids & Organic acid metabolism & CHR vs HC \\
\hline Prostaglandin A1 & 2.815 & 0.029 & -2.141 & 0.705 & Fatty acids & Fatty acid metabolism & CHR vs HC \\
\hline Bortezomib & 1.004 & 0.029 & -1.273 & 0.507 & Others & Others & CHR vs HC \\
\hline Deoxyuridine & 1.685 & 0.031 & -1.380 & 0.629 & Nucleotides & Pyrimidine metabolism & CHR vs HC \\
\hline Rigin & 1.076 & 0.032 & -1.358 & 0.605 & Peptides & Amino acid metabolism & CHR vs HC \\
\hline Ceramide (d18:1/16:0) & 1.280 & 0.032 & -1.199 & 0.614 & Lipids & Sphingolipid metabolism & CHR vs HC \\
\hline 6-Hydroxynicotinic acid & 1.957 & 0.032 & -1.393 & 0.659 & Others & $\begin{array}{l}\text { Nicotinate and nicotinamide } \\
\text { metabolism }\end{array}$ & CHR vs HC \\
\hline LysoPE 16:0 & 1.051 & 0.032 & -1.487 & 0.619 & Lipids & Lipid metabolism & CHR vs HC \\
\hline Dodecanedioic acid & 1.485 & 0.032 & -1.271 & 0.574 & Fatty acids & Fatty acid metabolism & CHR vs HC \\
\hline Ethyl glucuronide & 1.346 & 0.032 & -1.260 & 0.597 & Carbohydrates & Carbohydrate metabolism & CHR vs HC \\
\hline
\end{tabular}




\begin{tabular}{|c|c|c|c|c|c|c|c|}
\hline L-Arabinose & 1.095 & 0.032 & -1.195 & 0.526 & Carbohydrates & $\begin{array}{l}\text { Ascorbate and aldarate } \\
\text { metabolism }\end{array}$ & CHR vs HC \\
\hline (3-oxo-1-phenylbutoxy)sulfonic acid & 1.679 & 0.033 & -1.719 & 0.574 & Benzene derivatives & Benzene metabolism & CHR vs $\mathrm{HC}$ \\
\hline (S,S)-Nt-Histidinylalanine & 1.217 & 0.034 & 1.778 & 0.596 & Amino acids & Amino acid metabolism & CHR vs HC \\
\hline N-Heptanoylglycine & 1.343 & 0.034 & -1.259 & 0.638 & Amino acids & Amino acid metabolism & CHR vs HC \\
\hline Tyrosol 4-sulfate & 1.693 & 0.034 & -1.407 & 0.571 & Others & Others & CHR vs HC \\
\hline Orotidine & 1.293 & 0.034 & -1.433 & 0.615 & Nucleotides & Pyrimidine metabolism & CHR vs HC \\
\hline $3 \beta$-cholic acid & 1.242 & 0.035 & -1.135 & 0.589 & Lipids & Lipid metabolism & CHR vs HC \\
\hline 2-Hydroxylauric acid & 1.204 & 0.035 & -1.087 & 0.582 & Fatty acids & Fatty acid metabolism & CHR vs HC \\
\hline Succinic acid & 1.417 & 0.036 & 1.196 & 0.600 & Organic acids & Citrate cycle (TCA cycle) & CHR vs $\mathrm{HC}$ \\
\hline $\begin{array}{l}\text { D-Galactopyranosyl-(1->3)-D-galactopyranosyl- } \\
(1->3) \text {-L-arabinose }\end{array}$ & 1.407 & 0.036 & -1.896 & 0.598 & Carbohydrates & Carbohydrate metabolism & CHR vs HC \\
\hline Aspartyllysine & 1.178 & 0.036 & -2.733 & 0.657 & Peptides & Amino acid metabolism & CHR vs HC \\
\hline D-Ornithine & 1.141 & 0.037 & 1.301 & 0.624 & Amino acids & $\begin{array}{l}\text { D-Arginine and D-ornithine } \\
\text { metabolism }\end{array}$ & CHR vs HC \\
\hline$( \pm)$-Sulfobutanedioic acid & 1.305 & 0.037 & -1.259 & 0.587 & Lipids & Lipid metabolism & CHR vs HC \\
\hline $\begin{array}{l}\text { 3-(4-hydroxy-3,5-dimethoxyphenyl)oxirane-2- } \\
\text { carboxylic acid }\end{array}$ & 1.436 & 0.037 & -1.459 & 0.591 & Others & Others & CHR vs $\mathrm{HC}$ \\
\hline $\mathrm{C} 23: 0$ & 1.099 & 0.038 & -1.312 & 0.577 & Fatty acids & Fatty acid metabolism & CHR vs $\mathrm{HC}$ \\
\hline Histidinyl-Histidine & 1.388 & 0.042 & 1.024 & 0.523 & Peptides & Amino acid metabolism & CHR vs HC \\
\hline Dehydroascorbic acid & 1.661 & 0.042 & 1.203 & 0.623 & Others & Glutathione metabolism & CHR vs HC \\
\hline Methyl 1-(methylthio)propyl disulfide & 1.125 & 0.044 & -1.726 & 0.673 & Others & Others & CHR vs HC \\
\hline Acetoacetic acid & 1.892 & 0.044 & 2.069 & 0.680 & Organic acids & $\begin{array}{l}\text { Synthesis and degradation of } \\
\text { ketone bodies }\end{array}$ & CHR vs HC \\
\hline Vanillylmandelic acid & 1.725 & 0.044 & -1.456 & 0.597 & Benzene derivatives & Tyrosine metabolism & CHR vs HC \\
\hline 3-Methoxytyrosine & 1.129 & 0.044 & -1.097 & 0.543 & Amino acids & Amino acid metabolism & CHR vs $\mathrm{HC}$ \\
\hline
\end{tabular}




\begin{tabular}{|c|c|c|c|c|c|c|c|}
\hline Lycoperdic acid & 1.843 & 0.045 & 1.550 & 0.645 & Amino acids & Amino acid metabolism & CHR vs HC \\
\hline Dimethylguanosine & 1.278 & 0.046 & -1.447 & 0.642 & Nucleotides & Nucleotide metabolism & CHR vs HC \\
\hline dTDP-D-glucose & 1.833 & 0.048 & -1.304 & 0.620 & Nucleotides & Streptomycin biosynthesis & CHR vs HC \\
\hline Gluconolactone & 1.075 & 0.049 & -1.365 & 0.532 & Carbohydrates & Pentose phosphate pathway & CHR vs HC \\
\hline$(2 \mathrm{R}, 3 \mathrm{~S})$-Piscidic acid & 1.118 & 0.050 & -1.229 & 0.544 & Benzene derivatives & Benzene metabolism & CHR vs HC \\
\hline Sodium Tetradecyl Sulfate & 3.618 & 0.017 & 3.447 & 0.732 & Others & Others & FES vs CHR \\
\hline Saccharin & 3.385 & 0.022 & 6.324 & 0.681 & Benzene derivatives & Tsate transduction & FES vs CHR \\
\hline 4-OH-Retinal & 2.529 & 0.022 & -2.117 & 0.681 & Lipids & Lipid metabolism & FES vs CHR \\
\hline Squamosinin A & 3.506 & 0.022 & 2.735 & 0.730 & Lipids & Lipid metabolism & FES vs CHR \\
\hline CE(20:3(5Z,8Z,11Z)) & 1.842 & 0.022 & -1.639 & 0.674 & Lipids & Lipid metabolism & FES vs CHR \\
\hline Aniline-2-sulfonic acid & 2.263 & 0.023 & -1.494 & 0.672 & Others & Others & FES vs CHR \\
\hline Linalyl caprylate & 2.506 & 0.023 & -1.780 & 0.703 & Lipids & Lipid metabolism & FES vs CHR \\
\hline 5a-Tetrahydrocortisol & 2.991 & 0.023 & 1.444 & 0.596 & Lipids & Lipid metabolism & FES vs CHR \\
\hline Cerebronic acid & 3.082 & 0.023 & 1.922 & 0.742 & Fatty acids & Fatty acid metabolism & FES vs CHR \\
\hline Prenyl glucoside & 3.114 & 0.028 & 2.174 & 0.715 & Lipids & Lipid metabolism & FES vs CHR \\
\hline Lycoperdic acid & 2.517 & 0.028 & 1.170 & 0.621 & Amino acids & Amino acid metabolism & FES vs CHR \\
\hline Diethylhexyl adipate & 2.879 & 0.028 & 1.648 & 0.675 & Lipids & Lipid metabolism & FES vs CHR \\
\hline Glutamic acid & 2.295 & 0.028 & 1.628 & 0.642 & Amino acids & Glutathione metabolism & FES vs CHR \\
\hline Salicylamide & 1.461 & 0.028 & -1.305 & 0.596 & Benzene derivatives & Benzene metabolism & FES vs CHR \\
\hline Tridecanoic acid & 2.136 & 0.032 & -1.438 & 0.616 & Fatty acids & Fatty acid metabolism & FES vs CHR \\
\hline dTDP-D-glucose & 2.098 & 0.032 & -1.260 & 0.609 & Nucleotides & Streptomycin biosynthesis & FES vs CHR \\
\hline N5-Acetyl-N2-gamma-L-glutamyl-L-ornithine & 1.583 & 0.032 & 3.093 & 0.681 & Peptides & Amino acid metabolism & FES vs CHR \\
\hline C20:2(cis-11,14) & 1.811 & 0.032 & -1.668 & 0.630 & Fatty acids & $\begin{array}{l}\text { Biosynthesis of unsaturated } \\
\text { fatty acids }\end{array}$ & FES vs CHR \\
\hline Phaseolic acid & 2.822 & 0.033 & 3.783 & 0.703 & Organic acids & Organic acid metabolism & FES vs CHR \\
\hline Junicedral & 2.081 & 0.033 & -1.537 & 0.689 & Lipids & Lipid metabolism & FES vs CHR \\
\hline
\end{tabular}




\begin{tabular}{|c|c|c|c|c|c|c|c|}
\hline APGPR Enterostatin & 2.430 & 0.033 & -2.640 & 0.724 & Peptides & Amino acid metabolism & FES vs CHR \\
\hline Fumarycarnitine & 2.058 & 0.033 & 1.328 & 0.652 & Lipids & Lipid metabolism & FES vs CHR \\
\hline (S)-5-Diphosphomevalonic acid & 2.295 & 0.033 & 1.618 & 0.687 & Organic acids & $\begin{array}{l}\text { Biosynthesis of terpenoids } \\
\text { and steroids }\end{array}$ & FES vs CHR \\
\hline Hydroxyprolyl-Valine & 2.141 & 0.033 & 3.303 & 0.746 & Peptides & Amino acid metabolism & FES vs CHR \\
\hline cis-5-Tetradecenoylcarnitine & 1.750 & 0.034 & -1.573 & 0.758 & Lipids & Lipid metabolism & FES vs CHR \\
\hline Ethyl glucuronide & 1.741 & 0.034 & -1.060 & 0.556 & Carbohydrates & Carbohydrate metabolism & FES vs CHR \\
\hline $\begin{array}{l}\text { 3-[4-methoxy-3-(sulfooxy)phenyl]propanoic } \\
\text { acid }\end{array}$ & 2.128 & 0.034 & -1.982 & 0.595 & Benzene derivatives & Benzene metabolism & FES vs CHR \\
\hline 5'-Carboxy-gamma-chromanol & 2.126 & 0.034 & -2.045 & 0.559 & Others & Others & FES vs CHR \\
\hline Methyl 1-(methylthio)propyl disulfide & 2.386 & 0.039 & -1.805 & 0.655 & Others & Others & FES vs CHR \\
\hline Methyl beta-D-galactoside & 1.763 & 0.039 & -1.186 & 0.640 & Carbohydrates & Carbohydrate metabolism & FES vs CHR \\
\hline $\begin{array}{l}\text { 3,4,5-trihydroxy-6-(2-hydroxyethoxy)oxane-2- } \\
\text { carboxylic acid }\end{array}$ & 1.914 & 0.039 & -1.488 & 0.602 & Carbohydrates & Carbohydrate metabolism & FES vs CHR \\
\hline $\begin{array}{l}\text { [4-(5,7-dihydroxy-6,8-dimethyl-4-oxo-3,4- } \\
\text { dihydro-2H-1-benzopyran-2- } \\
\text { yl)phenyl]oxidanesulfonic acid }\end{array}$ & 1.726 & 0.039 & -1.424 & 0.573 & Others & Others & FES vs CHR \\
\hline O-Acetyl-L-homoserine & 1.225 & 0.041 & -1.276 & 0.570 & Amino acids & $\begin{array}{l}\text { Cysteine and methionine } \\
\text { metabolism }\end{array}$ & FES vs CHR \\
\hline Aesculetin & 1.962 & 0.041 & -1.550 & 0.575 & Others & $\begin{array}{l}\text { Biosynthesis of } \\
\text { phenylpropanoids }\end{array}$ & FES vs CHR \\
\hline $\operatorname{Cer}(\mathrm{d} 18: 1 / 14: 0)$ & 2.088 & 0.041 & -1.552 & 0.687 & Lipids & Lipid metabolism & FES vs CHR \\
\hline $\begin{array}{l}\text { Methyl 3-(2,3-dihydroxy-3-methylbutyl)-4- } \\
\text { hydroxybenzoate }\end{array}$ & 1.615 & 0.041 & -1.222 & 0.615 & Benzene derivatives & Benzene metabolism & FES vs CHR \\
\hline Prostaglandin D2 & 2.151 & 0.041 & -1.355 & 0.600 & Lipids & $\begin{array}{l}\text { Arachidonic acid } \\
\text { metabolism }\end{array}$ & FES vs CHR \\
\hline
\end{tabular}




\begin{tabular}{|c|c|c|c|c|c|c|c|}
\hline 9Z-Eicosenoic acid & 1.797 & 0.041 & -1.288 & 0.631 & Fatty acids & Fatty acid metabolism & FES vs CHR \\
\hline S-carboxymethyl-L-cysteine & 2.159 & 0.041 & 1.298 & 0.627 & Amino acids & Amino acid metabolism & FES vs CHR \\
\hline $\begin{array}{l}\text { 5-hydroxy-4-(4-hydroxyphenyl)-8,8-dimethyl- } \\
\text { 2H,8H-pyrano[2,3-f]chromen-2-one }\end{array}$ & 1.556 & 0.041 & 1.799 & 0.704 & Others & Others & FES vs CHR \\
\hline Ophthalmic acid & 1.250 & 0.041 & 2.780 & 0.686 & Peptides & Amino acid metabolism & FES vs CHR \\
\hline $\begin{array}{l}\text { [6-hydroxy-2-methoxy-3-(3-phenylprop-2-en-1- } \\
\text { yl)phenyl]oxidanesulfonic acid }\end{array}$ & 1.706 & 0.041 & -1.499 & 0.611 & Others & Others & FES vs CHR \\
\hline IDP & 1.059 & 0.041 & -1.173 & 0.585 & Nucleotides & Purine metabolism & FES vs CHR \\
\hline isodeoxycholic acid(isoDCA) & 1.100 & 0.041 & -1.096 & 0.539 & Lipids & Lipid metabolism & FES vs CHR \\
\hline Tyrosol 4-sulfate & 1.643 & 0.042 & -1.440 & 0.564 & Others & Others & FES vs CHR \\
\hline Nopalinic acid & 2.122 & 0.042 & 1.493 & 0.659 & Amino acids & Amino acid metabolism & FES vs CHR \\
\hline $\operatorname{MG}(0: 0 / 14: 0 / 0: 0)$ & 1.646 & 0.042 & -1.374 & 0.568 & Lipids & Lipid metabolism & FES vs CHR \\
\hline 5-Hydroxy-2-furoic acid & 1.384 & 0.042 & -1.201 & 0.551 & Others & Others & FES vs CHR \\
\hline $\begin{array}{l}\text { a-L-threo-4-Hex-4-enopyranuronosyl-D- } \\
\text { galacturonic acid }\end{array}$ & 2.069 & 0.043 & 1.108 & 0.629 & Carbohydrates & Carbohydrate metabolism & FES vs CHR \\
\hline PS(18:0/18:1(9Z)) & 1.436 & 0.043 & -1.246 & 0.597 & Lipids & $\begin{array}{l}\text { Glycerophospholipid } \\
\text { metabolism }\end{array}$ & FES vs CHR \\
\hline PE(P-16:0/20:4(5Z,8Z,11Z,14Z)) & 1.474 & 0.044 & -1.649 & 0.649 & Lipids & Lipid metabolism & FES vs CHR \\
\hline 5-hydroxyindoleacetic acid & 2.002 & 0.046 & 1.161 & 0.652 & Indoles & Tryptophan metabolism & FES vs CHR \\
\hline PS(18:2(9Z,12Z)/18:0) & 1.350 & 0.046 & -1.442 & 0.581 & Lipids & Lipid metabolism & FES vs CHR \\
\hline 4-Acetamido-2-aminobutanoic acid & 2.072 & 0.047 & 2.314 & 0.657 & Amino acids & $\begin{array}{l}\text { Glycine, serine and } \\
\text { threonine metabolism }\end{array}$ & FES vs CHR \\
\hline Homogentisic acid & 1.507 & 0.048 & -1.505 & 0.548 & Organic acids & Tyrosine metabolism & FES vs CHR \\
\hline N-Acetylthreonine & 1.537 & 0.048 & 1.870 & 0.675 & Amino acids & Amino acid metabolism & FES vs CHR \\
\hline Aprobarbital & 1.963 & 0.049 & -1.185 & 0.662 & Others & Others & FES vs CHR \\
\hline Indole-3-methyl acetate & 2.163 & 0.049 & -1.211 & 0.645 & Indoles & Indole metabolism & FES vs CHR \\
\hline
\end{tabular}


Prostaglandin A1

1.861

0.049

1.135

0.527 Fatty acids

Fatty acid metabolism

FES vs CHR

${ }^{a}$ False discovery rate adjusted q-values were calculated based on $\mathrm{p}$-values estimated by linear regression, adjusting for age, sex and the education level. 
Table S2. The diseases and biological functions in CHR subjects.

\begin{tabular}{|c|c|c|}
\hline Categories & Diseases or Functions Annotation & p-value \\
\hline Inflammatory Response, Neurological Disease & $\begin{array}{l}\text { Inflammation of central nervous } \\
\text { system }\end{array}$ & $1.63 \mathrm{E}-06$ \\
\hline $\begin{array}{l}\text { Inflammatory Disease, Inflammatory } \\
\text { Response, Neurological Disease, Organismal } \\
\text { Injury and Abnormalities }\end{array}$ & Encephalomyelitis & $5.85 \mathrm{E}-06$ \\
\hline $\begin{array}{l}\text { Hematological Disease, Immunological } \\
\text { Disease, Inflammatory Disease }\end{array}$ & Eosinophilic inflammation & $1.61 \mathrm{E}-05$ \\
\hline $\begin{array}{l}\text { Immunological Disease, Inflammatory } \\
\text { Disease, Inflammatory Response, } \\
\text { Neurological Disease, Organismal Injury and } \\
\text { Abnormalities }\end{array}$ & $\begin{array}{l}\text { Experimental autoimmune } \\
\text { encephalomyelitis }\end{array}$ & 0.000034 \\
\hline $\begin{array}{l}\text { Immunological Disease, Inflammatory } \\
\text { Disease, Inflammatory Response, } \\
\text { Neurological Disease, Organismal Injury and } \\
\text { Abnormalities }\end{array}$ & $\begin{array}{l}\text { Chronic phase experimental } \\
\text { autoimmune encephalomyelitis }\end{array}$ & $9.42 \mathrm{E}-05$ \\
\hline
\end{tabular}


Table S3. The correlation network of salivary metabolites with symptoms of two disease statuses.

\begin{tabular}{|c|c|c|c|c|c|c|}
\hline Severity & Metabolites & $\mathbf{R}$ & p-value & Classification & Pathway & Comparison \\
\hline BPRS & $\begin{array}{l}\text { a-L-threo-4-Hex-4-enopyranuronosyl-D- } \\
\text { galacturonic acid }\end{array}$ & -0.318 & $1.710 \mathrm{E}-02$ & Carbohydrates & Carbohydrate metabolism & FES vs HC \\
\hline BPRS & Asparaginyl-Tyrosine & 0.334 & $1.198 \mathrm{E}-02$ & Peptides & Amino acid metabolism & FES vs HC \\
\hline BPRS & Caryoptosidic acid & 0.315 & $1.820 \mathrm{E}-02$ & Lipids & Lipid metabolism & FES vs HC \\
\hline BPRS & $\operatorname{Cer}(\mathrm{d} 18: 1 / 14: 0)$ & 0.442 & $6.535 \mathrm{E}-04$ & Lipids & Lipid metabolism & FES vs HC \\
\hline BPRS & Glutaminyltyrosine & 0.405 & $1.956 \mathrm{E}-03$ & Peptides & Amino acid metabolism & FES vs HC \\
\hline BPRS & Glycyltyrosine & 0.302 & $2.371 \mathrm{E}-02$ & Peptides & Amino acid metabolism & FES vs HC \\
\hline BPRS & Lactic acid & 0.316 & $1.750 \mathrm{E}-02$ & Organic acids & Glycolysis / Gluconeogenesis & FES vs HC \\
\hline BPRS & Muricatacin & -0.317 & $1.714 \mathrm{E}-02$ & Others & Others & FES vs HC \\
\hline BPRS & N-Caffeoyltryptophan & -0.307 & $2.139 \mathrm{E}-02$ & Amino acids & Amino acid metabolism & FES vs HC \\
\hline BPRS & Norepinephrine sulfate & -0.295 & 2.713E-02 & Benzene derivatives & Tyrosine metabolism & FES vs HC \\
\hline BPRS & p-Octopamine & 0.282 & $3.557 \mathrm{E}-02$ & Others & Neuroactive ligand-receptor interaction & FES vs HC \\
\hline BPRS & Prostaglandin A1 & -0.321 & $1.580 \mathrm{E}-02$ & Fatty acids & Fatty acid metabolism & FES vs HC \\
\hline BPRS & Squamosinin A & -0.332 & $1.253 \mathrm{E}-02$ & Lipids & Lipid metabolism & FES vs HC \\
\hline BPRS & $\begin{array}{l}\text { Vanillylmandelic acid } \\
\text { (3beta,17alpha,23S,24S)-17,23-Epoxy- }\end{array}$ & -0.273 & 4.195E-02 & Benzene derivatives & Tyrosine metabolism & FES vs HC \\
\hline BPRS & $\begin{array}{l}\text { 3,24,29-trihydroxy-27-norlanost-8-en-15- } \\
\text { one }\end{array}$ & 0.335 & $1.149 \mathrm{E}-02$ & Lipids & Lipid metabolism & FES vs HC \\
\hline BPRS & (S,S)-Nt-Histidinylalanine & 0.368 & $5.202 \mathrm{E}-03$ & Amino acids & Amino acid metabolism & FES vs HC \\
\hline BPRS & 2-Aminophenol & -0.434 & $8.240 \mathrm{E}-04$ & Benzene derivatives & Tryptophan metabolism & FES vs HC \\
\hline BPRS & 2-Hydroxypyridine & -0.447 & 5.456E-04 & Nucleotides & Nucleotide metabolism & FES vs HC \\
\hline BPRS & 2-methyl-3-(sulfooxy)propanoic acid & -0.385 & $3.358 \mathrm{E}-03$ & Organic acids & Organic acid metabolism & FES vs HC \\
\hline BPRS & 3-Hydroxymethylglutaric acid & -0.461 & $3.476 \mathrm{E}-04$ & Organic acids & Valine, leucine and isoleucine degradation & FES vs HC \\
\hline
\end{tabular}




\begin{tabular}{|c|c|c|c|c|c|c|}
\hline BPRS & 5'-Carboxy-gamma-chromanol & -0.363 & $6.031 \mathrm{E}-03$ & Others & Others & FES vs HC \\
\hline CGI & Caryoptosidic acid & 0.232 & $4.556 \mathrm{E}-02$ & Lipids & Lipid metabolism & FES vs HC \\
\hline CGI & Glutamylalanine & -0.235 & $4.270 \mathrm{E}-02$ & Peptides & Amino acid metabolism & FES vs HC \\
\hline SANS & Carnosine & -0.319 & $1.659 \mathrm{E}-02$ & Peptides & Histidine metabolism & FES vs HC \\
\hline SANS & dUMP & -0.292 & $2.887 \mathrm{E}-02$ & Nucleotides & Pyrimidine metabolism & FES vs HC \\
\hline SANS & gamma-Glutamylisoleucine & -0.276 & $3.965 \mathrm{E}-02$ & Peptides & Amino acid metabolism & FES vs HC \\
\hline SANS & L-beta-aspartyl-L-alanine & -0.382 & $3.689 \mathrm{E}-03$ & Peptides & Amino acid metabolism & FES vs HC \\
\hline SANS & S-carboxymethyl-L-cysteine & -0.359 & $6.559 \mathrm{E}-03$ & Amino acids & Amino acid metabolism & FES vs HC \\
\hline SANS & 1-Naphthylamine & -0.299 & $2.543 \mathrm{E}-02$ & Others & Metabolism of xenobiotics by cytochrome P450 & FES vs HC \\
\hline $\begin{array}{l}\text { Affective } \\
\text { flattening }\end{array}$ & S-carboxymethyl-L-cysteine & -0.272 & $4.270 \mathrm{E}-02$ & Amino acids & Amino acid metabolism & FES vs HC \\
\hline $\begin{array}{l}\text { Affective } \\
\text { flattening }\end{array}$ & 5-hydroxyindoleacetic acid & 0.272 & 4.271E-02 & Indoles & Tryptophan metabolism & FES vs HC \\
\hline Alogia & L-beta-aspartyl-L-alanine & -0.442 & $6.555 \mathrm{E}-04$ & Peptides & Amino acid metabolism & FES vs HC \\
\hline Alogia & Serylserine & -0.267 & $4.685 \mathrm{E}-02$ & Peptides & Amino acid metabolism & FES vs HC \\
\hline Alogia & Trihydroxycoprostanoic acid & 0.286 & $3.272 \mathrm{E}-02$ & Lipids & Lipid metabolism & FES vs HC \\
\hline Alogia & 24,25-Dihydroxyvitamin D & 0.269 & $4.468 \mathrm{E}-02$ & Others & Others & FES vs HC \\
\hline Anhedonia & Alanyl-Glutamine & -0.298 & $2.552 \mathrm{E}-02$ & Peptides & Amino acid metabolism & FES vs HC \\
\hline Anhedonia & Alanyl-Serine & -0.325 & $1.438 \mathrm{E}-02$ & Peptides & Amino acid metabolism & FES vs HC \\
\hline Anhedonia & Asparaginyl-Tyrosine & -0.289 & $3.071 \mathrm{E}-02$ & Peptides & Amino acid metabolism & FES vs HC \\
\hline Anhedonia & Carnosine & -0.336 & $1.135 \mathrm{E}-02$ & Peptides & Histidine metabolism & FES vs HC \\
\hline Anhedonia & Histidinyl-Phenylalanine & -0.383 & $3.584 \mathrm{E}-03$ & Peptides & Amino acid metabolism & FES vs HC \\
\hline Anhedonia & L-beta-aspartyl-L-alanine & -0.331 & $1.283 \mathrm{E}-02$ & Peptides & Amino acid metabolism & FES vs HC \\
\hline Anhedonia & S-carboxymethyl-L-cysteine & -0.310 & $1.992 \mathrm{E}-02$ & Amino acids & Amino acid metabolism & FES vs HC \\
\hline Anhedonia & Serylserine & -0.333 & $1.226 \mathrm{E}-02$ & Peptides & Amino acid metabolism & FES vs HC \\
\hline Anhedonia & Tyrosyl-Isoleucine & -0.281 & $3.602 \mathrm{E}-02$ & Peptides & Amino acid metabolism & FES vs HC \\
\hline
\end{tabular}




\begin{tabular}{|c|c|c|c|c|c|c|}
\hline Anhedonia & $\begin{array}{l}\text { 1-Naphthylamine } \\
\text { (3beta,17alpha,23S,24S)-17,23-Epoxy- }\end{array}$ & -0.334 & $1.200 \mathrm{E}-02$ & Others & Metabolism of xenobiotics by cytochrome P450 & FES vs HC \\
\hline Attention & $\begin{array}{l}\text { 3,24,29-trihydroxy-27-norlanost-8-en-15- } \\
\text { one }\end{array}$ & 0.385 & $3.419 \mathrm{E}-03$ & Lipids & Lipid metabolism & FES vs HC \\
\hline Avolition & Alanyl-Glutamine & -0.290 & $2.998 \mathrm{E}-02$ & Peptides & Amino acid metabolism & FES vs HC \\
\hline Avolition & Carnosine & -0.307 & $2.126 \mathrm{E}-02$ & Peptides & Histidine metabolism & FES vs HC \\
\hline Avolition & Histidinyl-Phenylalanine & -0.317 & $1.732 \mathrm{E}-02$ & Peptides & Amino acid metabolism & FES vs HC \\
\hline Avolition & L-beta-aspartyl-L-alanine & -0.370 & $5.033 \mathrm{E}-03$ & Peptides & Amino acid metabolism & FES vs HC \\
\hline Avolition & S-carboxymethyl-L-cysteine & -0.279 & $3.715 \mathrm{E}-02$ & Amino acids & Amino acid metabolism & FES vs HC \\
\hline Avolition & Serylserine & -0.283 & $3.472 \mathrm{E}-02$ & Peptides & Amino acid metabolism & FES vs HC \\
\hline DS & Hydroquinone & -0.397 & $1.237 \mathrm{E}-02$ & Benzene derivatives & Tyrosine metabolism & CHR vs HC \\
\hline DS & Junicedral & 0.380 & $1.699 \mathrm{E}-02$ & Lipids & Lipid metabolism & CHR vs HC \\
\hline GS & L-Cyclo(alanylglycyl) & -0.334 & $3.791 \mathrm{E}-02$ & Others & Others & CHR vs HC \\
\hline GS & Metronidazole & -0.349 & $2.938 \mathrm{E}-02$ & Others & Others & CHR vs HC \\
\hline GS & Pyridoxic acid & -0.354 & 2.694E-02 & Others & Vitamin B6 metabolism & CHR vs HC \\
\hline GS & 5-L-Glutamyl-taurine & -0.439 & $5.185 \mathrm{E}-03$ & Amino acids & Taurine and hypotaurine metabolism & CHR vs HC \\
\hline NS & Acetoin & -0.351 & $2.832 \mathrm{E}-02$ & Others & Others & CHR vs HC \\
\hline NS & Aniline-2-sulfonic acid & -0.409 & $9.654 \mathrm{E}-03$ & Others & Others & CHR vs HC \\
\hline NS & Citrulline & 0.430 & $6.227 \mathrm{E}-03$ & Amino acids & Arginine biosynthesis & CHR vs HC \\
\hline NS & Cortisol & -0.364 & $2.292 \mathrm{E}-02$ & Lipids & Steroid hormone biosynthesis & CHR vs HC \\
\hline NS & D-Ornithine & 0.420 & $7.836 \mathrm{E}-03$ & Amino acids & D-Arginine and D-ornithine metabolism & CHR vs HC \\
\hline NS & Dimethylguanosine & -0.378 & $1.776 \mathrm{E}-02$ & Nucleotides & Nucleotide metabolism & CHR vs HC \\
\hline NS & Dodecanedioic acid & -0.350 & $2.906 \mathrm{E}-02$ & Fatty acids & Fatty acid metabolism & CHR vs HC \\
\hline NS & dTDP-D-glucose & -0.462 & $3.099 \mathrm{E}-03$ & Nucleotides & Streptomycin biosynthesis & CHR vs HC \\
\hline NS & Ethyl beta-D-glucopyranoside & -0.334 & $3.741 \mathrm{E}-02$ & Carbohydrates & Carbohydrate metabolism & CHR vs HC \\
\hline NS & Gluconolactone & -0.399 & $1.193 \mathrm{E}-02$ & Carbohydrates & Pentose phosphate pathway & CHR vs HC \\
\hline
\end{tabular}




\begin{tabular}{|c|c|c|c|c|c|c|}
\hline NS & $\begin{array}{l}\text { Glucosyl (2E,6E,10x)-10,11-dihydroxy-2,6- } \\
\text { farnesadienoate }\end{array}$ & -0.370 & $2.030 \mathrm{E}-02$ & Lipids & Lipid metabolism & CHR vs HC \\
\hline NS & Glucosylisomaltol & -0.471 & $2.457 \mathrm{E}-03$ & Carbohydrates & Carbohydrate metabolism & CHR vs HC \\
\hline NS & Homogentisic acid & -0.361 & $2.406 \mathrm{E}-02$ & Organic acids & Tyrosine metabolism & CHR vs HC \\
\hline NS & Lysyl-Serine & 0.321 & $4.631 \mathrm{E}-02$ & Peptides & Amino acid metabolism & CHR vs HC \\
\hline NS & Methyl 1-(methylthio)propyl disulfide & -0.319 & $4.747 \mathrm{E}-02$ & Others & Others & CHR vs HC \\
\hline NS & Orotidine & -0.325 & 4.342E-02 & Nucleotides & Pyrimidine metabolism & CHR vs $\mathrm{HC}$ \\
\hline NS & p-Aminobenzoic acid & -0.349 & $2.930 \mathrm{E}-02$ & Benzene derivatives & Aminobenzoate degradation & CHR vs HC \\
\hline NS & Phaseolic acid & -0.363 & $2.327 \mathrm{E}-02$ & Organic acids & Organic acid metabolism & CHR vs HC \\
\hline NS & Prenyl glucoside & -0.387 & $1.504 \mathrm{E}-02$ & Lipids & Lipid metabolism & CHR vs HC \\
\hline NS & Prolyl-Glutamine & 0.347 & $3.020 \mathrm{E}-02$ & Peptides & Amino acid metabolism & CHR vs HC \\
\hline NS & Pyridoxic acid & -0.406 & $1.027 \mathrm{E}-02$ & Others & Vitamin B6 metabolism & CHR vs HC \\
\hline NS & Tyrosol 4-sulfate & -0.361 & $2.389 \mathrm{E}-02$ & Others & Others & CHR vs HC \\
\hline NS & Vanillylmandelic acid & -0.345 & $3.167 \mathrm{E}-02$ & Benzene derivatives & Tyrosine metabolism & CHR vs HC \\
\hline NS & [(oxolan-2-yl)methoxy]sulfonic acid & -0.393 & $1.328 \mathrm{E}-02$ & Others & Others & CHR vs HC \\
\hline NS & $(2 \mathrm{R}, 3 \mathrm{~S})$-Piscidic acid & -0.369 & $2.076 \mathrm{E}-02$ & Benzene derivatives & Benzene metabolism & CHR vs HC \\
\hline NS & (3-oxo-1-phenylbutoxy)sulfonic acid & -0.405 & $1.054 \mathrm{E}-02$ & Benzene derivatives & Benzene metabolism & CHR vs HC \\
\hline NS & $(\mathrm{S}, \mathrm{S})$-Nt-Histidinylalanine & 0.327 & $4.221 \mathrm{E}-02$ & Amino acids & Amino acid metabolism & CHR vs HC \\
\hline NS & 15-Deoxy-d-12,14-PGJ2 & -0.336 & $3.624 \mathrm{E}-02$ & Lipids & Arachidonic acid metabolism & CHR vs HC \\
\hline NS & 2-Aminophenol & -0.472 & $2.423 \mathrm{E}-03$ & Benzene derivatives & Tryptophan metabolism & CHR vs HC \\
\hline NS & 2-Hydroxylauric acid & -0.340 & $3.439 \mathrm{E}-02$ & Fatty acids & Fatty acid metabolism & CHR vs HC \\
\hline NS & 2-Hydroxypyridine & -0.438 & $5.269 \mathrm{E}-03$ & Nucleotides & Nucleotide metabolism & CHR vs HC \\
\hline NS & $\begin{array}{l}\text { 3-(4-hydroxy-3,5-dimethoxyphenyl)oxirane- } \\
\text { 2-carboxylic acid }\end{array}$ & -0.408 & $9.905 \mathrm{E}-03$ & Others & Others & CHR vs HC \\
\hline NS & 3,4-Methylenesebacic acid & -0.371 & $1.992 \mathrm{E}-02$ & Fatty acids & Fatty acid metabolism & CHR vs HC \\
\hline NS & 3-Methylxanthine & -0.319 & $4.775 \mathrm{E}-02$ & Nucleotides & Caffeine metabolism & CHR vs HC \\
\hline
\end{tabular}




\begin{tabular}{|c|c|c|c|c|c|c|}
\hline NS & 5'-Carboxy-gamma-chromanol & -0.370 & $2.045 \mathrm{E}-02$ & Others & Others & CHR vs HC \\
\hline NS & $\begin{array}{l}\text { 6-hydroxy-7-methoxy-2H-1,3-benzodioxole- } \\
\text { 5-carboxylic acid }\end{array}$ & -0.399 & $1.185 \mathrm{E}-02$ & Benzene derivatives & Benzene metabolism & CHR vs HC \\
\hline NS & 6-Hydroxynicotinic acid & -0.365 & $2.230 \mathrm{E}-02$ & Others & Nicotinate and nicotinamide metabolism & CHR vs HC \\
\hline NS & 9E,11E-Octadecadienoic acid & -0.402 & $1.123 \mathrm{E}-02$ & Fatty acids & Fatty acid metabolism & CHR vs HC \\
\hline PS & Campesterol & 0.420 & $7.788 \mathrm{E}-03$ & Lipids & Steroid biosynthesis & CHR vs HC \\
\hline PS & 11-Dehydro-thromboxane B2 & 0.327 & 4.223E-02 & Lipids & Arachidonic acid metabolism & CHR vs HC \\
\hline PS & 4-OH-Retinal & 0.318 & 4.872E-02 & Lipids & Lipid metabolism & CHR vs HC \\
\hline SIPS & dTDP-D-glucose & -0.333 & $3.825 \mathrm{E}-02$ & Nucleotides & Streptomycin biosynthesis & CHR vs HC \\
\hline SIPS & $\begin{array}{l}\text { Glucosyl (2E,6E,10x)-10,11-dihydroxy-2,6- } \\
\text { farnesadienoate }\end{array}$ & -0.366 & $2.193 \mathrm{E}-02$ & Lipids & Lipid metabolism & CHR vs HC \\
\hline SIPS & Glucosylisomaltol & -0.361 & $2.410 \mathrm{E}-02$ & Carbohydrates & Carbohydrate metabolism & CHR vs HC \\
\hline SIPS & Phaseolic acid & -0.357 & $2.555 \mathrm{E}-02$ & Organic acids & Organic acid metabolism & CHR vs HC \\
\hline SIPS & Prenyl glucoside & -0.358 & $2.523 \mathrm{E}-02$ & Lipids & Lipid metabolism & CHR vs HC \\
\hline SIPS & Prolyl-Glutamine & 0.331 & $3.935 \mathrm{E}-02$ & Peptides & Amino acid metabolism & CHR vs HC \\
\hline SIPS & Pyridoxic acid & -0.443 & $4.738 \mathrm{E}-03$ & Others & Vitamin B6 metabolism & CHR vs HC \\
\hline
\end{tabular}


Table S4. The distinct relationships between salivary metabolites and blood markers in the FES and HC groups, respectively.

\begin{tabular}{|c|c|c|c|c|c|c|}
\hline Blood markers & Metabolites & $\mathbf{R}$ & p-value & Group & Classification & Pathway \\
\hline CRP & Vanillylmandelic acid & 0.455 & $1.476 \mathrm{E}-03$ & $\mathrm{HC}$ & Benzene derivatives & Tyrosine metabolism \\
\hline IL_8 & Urate radical & 0.295 & $4.627 \mathrm{E}-02$ & $\mathrm{HC}$ & Nucleotides & Nucleotide metabolism \\
\hline IL_8 & Uric acid & 0.305 & $3.909 \mathrm{E}-02$ & $\mathrm{HC}$ & Nucleotides & Purine metabolism \\
\hline Thioredoxin & Succinic acid & -0.329 & $2.552 \mathrm{E}-02$ & $\mathrm{HC}$ & Organic acids & Citrate cycle (TCA cycle) \\
\hline CRP & Urate radical & -0.412 & $6.646 \mathrm{E}-03$ & FES & Nucleotides & Nucleotide metabolism \\
\hline CRP & Uric acid & -0.379 & $1.343 \mathrm{E}-02$ & FES & Nucleotides & Purine metabolism \\
\hline $\mathrm{IFN} \gamma$ & Hydroquinone & -0.379 & $1.343 \mathrm{E}-02$ & FES & Benzene derivatives & Tyrosine metabolism \\
\hline IL_1 $1 \beta$ & Metanephrine & -0.308 & 4.693E-02 & FES & Benzene derivatives & Tyrosine metabolism \\
\hline IL_8 & 5-Hydroxyindoleacetaldehyde & 0.352 & $2.223 \mathrm{E}-02$ & FES & Indoles & Tryptophan metabolism \\
\hline IL_8 & Hydroquinone & -0.477 & $1.401 \mathrm{E}-03$ & FES & Benzene derivatives & Tyrosine metabolism \\
\hline IL_8 & Riboflavin & 0.338 & $2.847 \mathrm{E}-02$ & FES & Others & Riboflavin metabolism \\
\hline IL_8 & Succinic acid & -0.317 & 4.071E-02 & FES & Organic acids & Citrate cycle (TCA cycle) \\
\hline S100B & 2-Aminophenol & -0.326 & $3.514 \mathrm{E}-02$ & FES & Benzene derivatives & Tryptophan metabolism \\
\hline S100B & 5-hydroxyindoleacetic acid & -0.363 & $1.817 \mathrm{E}-02$ & FES & Indoles & Tryptophan metabolism \\
\hline S100B & Hydroquinone & -0.375 & $1.429 \mathrm{E}-02$ & FES & Benzene derivatives & Tyrosine metabolism \\
\hline S100B & Vanillylmandelic acid & -0.328 & 3.394E-02 & FES & Benzene derivatives & Tyrosine metabolism \\
\hline Thioredoxin & 2-Aminophenol & 0.361 & $1.883 \mathrm{E}-02$ & FES & Benzene derivatives & Tryptophan metabolism \\
\hline Thioredoxin & Acetoacetic acid & -0.353 & $2.166 \mathrm{E}-02$ & FES & Organic acids & Synthesis and degradation of ketone bodies \\
\hline Thioredoxin & Itaconic acid & -0.394 & $9.887 \mathrm{E}-03$ & FES & Fatty acids & C5-Branched dibasic acid metabolism \\
\hline Thioredoxin & L-2-hydroxyglutaric acid & -0.394 & $9.792 \mathrm{E}-03$ & FES & Organic acids & Lysine degradation \\
\hline Thioredoxin & Vanillylmandelic acid & 0.360 & $1.917 \mathrm{E}-02$ & FES & Benzene derivatives & Tyrosine metabolism \\
\hline $\mathrm{TNF} \alpha$ & Hydroquinone & -0.535 & $2.610 \mathrm{E}-04$ & FES & Benzene derivatives & Tyrosine metabolism \\
\hline $\mathrm{TNF} \alpha$ & Riboflavin & 0.433 & $4.196 \mathrm{E}-03$ & FES & Others & Riboflavin metabolism \\
\hline
\end{tabular}


Table S5. The correlations of salivary metabolites with salivary taxa.

\begin{tabular}{|c|c|c|c|c|c|c|}
\hline Microbiota & Phylum & Metabolites & Classification & Pathway & $\mathbf{R}$ & $\mathbf{p}$ \\
\hline Acidovorax & Proteobacteria & Dopamine 3-O-sulfate & Benzene derivatives & Tyrosine metabolism & 0.317 & $4.189 \mathrm{E}-03$ \\
\hline Actinobacillus & Proteobacteria & Orotidine & Nucleotides & Pyrimidine metabolism & 0.230 & 4.001E-02 \\
\hline Actinobacillus & Proteobacteria & riboflavin & Others & Riboflavin metabolism & 0.268 & $1.622 \mathrm{E}-02$ \\
\hline Actinomyces & Actinobacteria & Acetoacetic acid & Organic acids & $\begin{array}{l}\text { Synthesis and degradation of ketone } \\
\text { bodies }\end{array}$ & 0.224 & $4.545 \mathrm{E}-02$ \\
\hline Actinomyces & Actinobacteria & Glycine & Amino acids & Purine metabolism & 0.272 & $1.466 \mathrm{E}-02$ \\
\hline Actinomyces & Actinobacteria & L-Dopa & Amino acids & Tyrosine metabolism & -0.327 & $3.095 \mathrm{E}-03$ \\
\hline Actinomyces & Actinobacteria & Orotidine & Nucleotides & Pyrimidine metabolism & -0.367 & $8.265 \mathrm{E}-04$ \\
\hline Aggregatibacter & Proteobacteria & Acetoacetic acid & Organic acids & $\begin{array}{l}\text { Synthesis and degradation of ketone } \\
\text { bodies }\end{array}$ & 0.396 & $2.808 \mathrm{E}-04$ \\
\hline Aggregatibacter & Proteobacteria & Glutamine & Amino acids & Pyrimidine metabolism & 0.271 & $1.514 \mathrm{E}-02$ \\
\hline Aggregatibacter & Proteobacteria & Glycine & Amino acids & Purine metabolism & 0.256 & $2.179 \mathrm{E}-02$ \\
\hline Aggregatibacter & Proteobacteria & Hydroquinone & Benzene derivatives & Tyrosine metabolism & 0.230 & 4.037E-02 \\
\hline Aggregatibacter & Proteobacteria & Itaconic acid & Fatty acids & C5-Branched dibasic acid metabolism & 0.411 & $1.555 \mathrm{E}-04$ \\
\hline Aggregatibacter & Proteobacteria & L-2-hydroxyglutaric acid & Organic acids & Lysine degradation & 0.412 & $1.446 \mathrm{E}-04$ \\
\hline Aggregatibacter & Proteobacteria & Orotidine & Nucleotides & Pyrimidine metabolism & -0.287 & $9.773 \mathrm{E}-03$ \\
\hline Aggregatibacter & Proteobacteria & riboflavin & Others & Riboflavin metabolism & -0.275 & $1.341 \mathrm{E}-02$ \\
\hline Aggregatibacter & Proteobacteria & Urate radical & Nucleotides & Nucleotide metabolism & 0.268 & $1.621 \mathrm{E}-02$ \\
\hline Aggregatibacter & Proteobacteria & Uric acid & Nucleotides & Purine metabolism & 0.332 & $2.632 \mathrm{E}-03$ \\
\hline Atopobium & Actinobacteria & Isocitric acid & Organic acids & Citrate cycle (TCA cycle) & 0.422 & $9.580 \mathrm{E}-05$ \\
\hline Atopobium & Actinobacteria & riboflavin & Others & Riboflavin metabolism & -0.352 & $1.372 \mathrm{E}-03$ \\
\hline
\end{tabular}




\begin{tabular}{|c|c|c|c|c|c|c|}
\hline Blautia & Firmicutes & Acetoacetic acid & Organic acids & $\begin{array}{l}\text { Synthesis and degradation of ketone } \\
\text { bodies }\end{array}$ & 0.240 & $3.189 \mathrm{E}-02$ \\
\hline Blautia & Firmicutes & Itaconic acid & Fatty acids & C5-Branched dibasic acid metabolism & 0.257 & $2.123 \mathrm{E}-02$ \\
\hline Blautia & Firmicutes & L-2-hydroxyglutaric acid & Organic acids & Lysine degradation & 0.278 & $1.267 \mathrm{E}-02$ \\
\hline Brachybacterium & Actinobacteria & Isocitric acid & Organic acids & Citrate cycle (TCA cycle) & 0.242 & $3.087 \mathrm{E}-02$ \\
\hline Campylobacter & Proteobacteria & Normetanephrine & Benzene derivatives & Tyrosine metabolism & 0.273 & $1.425 \mathrm{E}-02$ \\
\hline Campylobacter & Proteobacteria & Urate radical & Nucleotides & Nucleotide metabolism & 0.245 & $2.829 \mathrm{E}-02$ \\
\hline Catonella & Firmicutes & 2-Aminophenol & Benzene derivatives & Tryptophan metabolism & 0.420 & $1.066 \mathrm{E}-04$ \\
\hline Catonella & Firmicutes & Hydroquinone & Benzene derivatives & Tyrosine metabolism & 0.297 & 7.411E-03 \\
\hline Catonella & Firmicutes & Isocitric acid & Organic acids & Citrate cycle (TCA cycle) & 0.469 & $1.120 \mathrm{E}-05$ \\
\hline Catonella & Firmicutes & Norepinephrine sulfate & Benzene derivatives & Tyrosine metabolism & 0.305 & $5.876 \mathrm{E}-03$ \\
\hline Catonella & Firmicutes & riboflavin & Others & Riboflavin metabolism & -0.319 & $3.926 \mathrm{E}-03$ \\
\hline Catonella & Firmicutes & Urate radical & Nucleotides & Nucleotide metabolism & 0.268 & $1.618 \mathrm{E}-02$ \\
\hline Catonella & Firmicutes & Vanillylmandelic acid & Benzene derivatives & Tyrosine metabolism & 0.427 & $7.660 \mathrm{E}-05$ \\
\hline Cupriavidus & Proteobacteria & Isocitric acid & Organic acids & Citrate cycle (TCA cycle) & 0.303 & $6.298 \mathrm{E}-03$ \\
\hline Cupriavidus & Proteobacteria & Orotidine & Nucleotides & Pyrimidine metabolism & 0.293 & $8.332 \mathrm{E}-03$ \\
\hline Cupriavidus & Proteobacteria & phenylacetylglycine & Amino acids & Phenylalanine metabolism & 0.228 & 4.164E-02 \\
\hline $\begin{array}{l}\text { Defluviitaleaceae } \\
\text { UCG-011 }\end{array}$ & Firmicutes & Glutamine & Amino acids & Pyrimidine metabolism & 0.221 & 4.899E-02 \\
\hline $\begin{array}{l}\text { Defluviitaleaceae } \\
\text { UCG-011 }\end{array}$ & Firmicutes & Isocitric acid & Organic acids & Citrate cycle (TCA cycle) & 0.356 & $1.184 \mathrm{E}-03$ \\
\hline $\begin{array}{l}\text { Defluviitaleaceae } \\
\text { UCG-011 }\end{array}$ & Firmicutes & riboflavin & Others & Riboflavin metabolism & -0.321 & $3.671 \mathrm{E}-03$ \\
\hline Desulfobulbus & Proteobacteria & Isocitric acid & Organic acids & Citrate cycle (TCA cycle) & 0.421 & $1.002 \mathrm{E}-04$ \\
\hline Desulfobulbus & Proteobacteria & riboflavin & Others & Riboflavin metabolism & -0.324 & $3.376 \mathrm{E}-03$ \\
\hline Desulfobulbus & Proteobacteria & Urate radical & Nucleotides & Nucleotide metabolism & 0.252 & 2.409E-02 \\
\hline
\end{tabular}




\begin{tabular}{|c|c|c|c|c|c|c|}
\hline Desulfobulbus & Proteobacteria & Uric acid & Nucleotides & Purine metabolism & 0.305 & $5.920 \mathrm{E}-03$ \\
\hline $\begin{array}{l}\text { Erysipelotrichaceae } \\
\text { UCG-006 }\end{array}$ & Firmicutes & phenylacetylglycine & Amino acids & Phenylalanine metabolism & 0.301 & 6.732E-03 \\
\hline $\begin{array}{l}\text { Eubacterium } \\
\text { nodatum group }\end{array}$ & Firmicutes & Acetoacetic acid & Organic acids & $\begin{array}{l}\text { Synthesis and degradation of ketone } \\
\text { bodies }\end{array}$ & 0.265 & $1.755 \mathrm{E}-02$ \\
\hline $\begin{array}{l}\text { Eubacterium } \\
\text { nodatum group }\end{array}$ & Firmicutes & Glycine & Amino acids & Purine metabolism & 0.265 & $1.762 \mathrm{E}-02$ \\
\hline $\begin{array}{l}\text { Eubacterium } \\
\text { nodatum group }\end{array}$ & Firmicutes & Itaconic acid & Fatty acids & C5-Branched dibasic acid metabolism & 0.312 & 4.822E-03 \\
\hline $\begin{array}{l}\text { Eubacterium } \\
\text { nodatum group }\end{array}$ & Firmicutes & L-2-hydroxyglutaric acid & Organic acids & Lysine degradation & 0.314 & $4.573 \mathrm{E}-03$ \\
\hline $\begin{array}{l}\text { Eubacterium } \\
\text { nodatum group }\end{array}$ & Firmicutes & L-Dopa & Amino acids & Tyrosine metabolism & -0.244 & $2.930 \mathrm{E}-02$ \\
\hline $\begin{array}{l}\text { Eubacterium } \\
\text { nodatum group }\end{array}$ & Firmicutes & Orotidine & Nucleotides & Pyrimidine metabolism & -0.250 & $2.532 \mathrm{E}-02$ \\
\hline $\begin{array}{l}\text { Eubacterium } \\
\text { nodatum group }\end{array}$ & Firmicutes & phenylacetylglycine & Amino acids & Phenylalanine metabolism & -0.227 & 4.305E-02 \\
\hline $\begin{array}{l}\text { Family XIII UCG- } \\
001\end{array}$ & Firmicutes & $\begin{array}{l}\text { 5- } \\
\text { Hydroxyindoleacetaldehyde }\end{array}$ & Indoles & Tryptophan metabolism & -0.228 & 4.193E-02 \\
\hline Fusobacterium & Fusobacteria & Isocitric acid & Organic acids & Citrate cycle (TCA cycle) & 0.369 & $7.446 \mathrm{E}-04$ \\
\hline Geobacillus & Firmicutes & Acetoacetic acid & Organic acids & $\begin{array}{l}\text { Synthesis and degradation of ketone } \\
\text { bodies }\end{array}$ & 0.339 & 2.103E-03 \\
\hline Geobacillus & Firmicutes & Itaconic acid & Fatty acids & C5-Branched dibasic acid metabolism & 0.351 & $1.393 \mathrm{E}-03$ \\
\hline Geobacillus & Firmicutes & L-2-hydroxyglutaric acid & Organic acids & Lysine degradation & 0.357 & $1.146 \mathrm{E}-03$ \\
\hline Geobacillus & Firmicutes & L-Dopa & Amino acids & Tyrosine metabolism & -0.256 & $2.190 \mathrm{E}-02$ \\
\hline Geobacillus & Firmicutes & Orotidine & Nucleotides & Pyrimidine metabolism & -0.259 & $2.030 \mathrm{E}-02$ \\
\hline
\end{tabular}




\begin{tabular}{|c|c|c|c|c|c|c|}
\hline Geobacillus & Firmicutes & Urate radical & Nucleotides & Nucleotide metabolism & 0.241 & $3.114 \mathrm{E}-02$ \\
\hline Geobacillus & Firmicutes & Uric acid & Nucleotides & Purine metabolism & 0.295 & 7.941E-03 \\
\hline Haemophilus & Proteobacteria & dUMP & Nucleotides & Pyrimidine metabolism & 0.346 & $1.664 \mathrm{E}-03$ \\
\hline Haemophilus & Proteobacteria & Glutamine & Amino acids & Pyrimidine metabolism & 0.243 & 2.969E-02 \\
\hline Haemophilus & Proteobacteria & Vanillylmandelic acid & Benzene derivatives & Tyrosine metabolism & -0.273 & $1.423 \mathrm{E}-02$ \\
\hline Hyphomicrobium & Proteobacteria & 2-Aminophenol & Benzene derivatives & Tryptophan metabolism & 0.231 & $3.963 \mathrm{E}-02$ \\
\hline Hyphomicrobium & Proteobacteria & 5-hydroxyindoleacetic acid & Indoles & Tryptophan metabolism & 0.289 & $9.446 \mathrm{E}-03$ \\
\hline Hyphomicrobium & Proteobacteria & Isocitric acid & Organic acids & Citrate cycle (TCA cycle) & 0.287 & $9.791 \mathrm{E}-03$ \\
\hline Hyphomicrobium & Proteobacteria & Orotidine & Nucleotides & Pyrimidine metabolism & 0.330 & $2.790 \mathrm{E}-03$ \\
\hline Johnsonella & Firmicutes & 5-hydroxyindoleacetic acid & Indoles & Tryptophan metabolism & 0.249 & $2.578 \mathrm{E}-02$ \\
\hline Johnsonella & Firmicutes & Isocitric acid & Organic acids & Citrate cycle (TCA cycle) & 0.254 & 2.297E-02 \\
\hline Kingella & Proteobacteria & Acetoacetic acid & Organic acids & $\begin{array}{l}\text { Synthesis and degradation of ketone } \\
\text { bodies }\end{array}$ & 0.256 & 2.187E-02 \\
\hline Kingella & Proteobacteria & Isocitric acid & Organic acids & Citrate cycle (TCA cycle) & 0.275 & $1.339 \mathrm{E}-02$ \\
\hline Kingella & Proteobacteria & Itaconic acid & Fatty acids & C5-Branched dibasic acid metabolism & 0.248 & $2.686 \mathrm{E}-02$ \\
\hline Kingella & Proteobacteria & L-2-hydroxyglutaric acid & Organic acids & Lysine degradation & 0.253 & $2.331 \mathrm{E}-02$ \\
\hline Kingella & Proteobacteria & riboflavin & Others & Riboflavin metabolism & -0.260 & $2.008 \mathrm{E}-02$ \\
\hline Kingella & Proteobacteria & Urate radical & Nucleotides & Nucleotide metabolism & 0.240 & $3.196 \mathrm{E}-02$ \\
\hline Kingella & Proteobacteria & Uric acid & Nucleotides & Purine metabolism & 0.325 & $3.243 \mathrm{E}-03$ \\
\hline Kingella & Proteobacteria & Vanillylmandelic acid & Benzene derivatives & Tyrosine metabolism & 0.243 & 2.982E-02 \\
\hline $\begin{array}{l}\text { Lachnospiraceae } \\
\text { NK4A136 group }\end{array}$ & Firmicutes & Acetoacetic acid & Organic acids & $\begin{array}{l}\text { Synthesis and degradation of ketone } \\
\text { bodies }\end{array}$ & 0.234 & $3.635 \mathrm{E}-02$ \\
\hline $\begin{array}{l}\text { Lachnospiraceae } \\
\text { NK4A136 group }\end{array}$ & Firmicutes & Itaconic acid & Fatty acids & C5-Branched dibasic acid metabolism & 0.271 & $1.523 \mathrm{E}-02$ \\
\hline $\begin{array}{l}\text { Lachnospiraceae } \\
\text { NK4A136 group }\end{array}$ & Firmicutes & L-2-hydroxyglutaric acid & Organic acids & Lysine degradation & 0.272 & $1.471 \mathrm{E}-02$ \\
\hline
\end{tabular}




\begin{tabular}{|c|c|c|c|c|c|c|}
\hline $\begin{array}{l}\text { Lachnospiraceae } \\
\text { NK4A136 group }\end{array}$ & Firmicutes & Normetanephrine & Benzene derivatives & Tyrosine metabolism & -0.236 & 3.497E-02 \\
\hline Leptotrichia & Fusobacteria & 2-Aminophenol & Benzene derivatives & Tryptophan metabolism & 0.531 & 4.040E-07 \\
\hline Leptotrichia & Fusobacteria & 5-hydroxyindoleacetic acid & Indoles & Tryptophan metabolism & 0.399 & $2.441 \mathrm{E}-04$ \\
\hline Leptotrichia & Fusobacteria & Glutamine & Amino acids & Pyrimidine metabolism & 0.235 & $3.601 \mathrm{E}-02$ \\
\hline Leptotrichia & Fusobacteria & Isocitric acid & Organic acids & Citrate cycle (TCA cycle) & 0.359 & $1.084 \mathrm{E}-03$ \\
\hline Leptotrichia & Fusobacteria & Norepinephrine sulfate & Benzene derivatives & Tyrosine metabolism & 0.280 & $1.179 \mathrm{E}-02$ \\
\hline Leptotrichia & Fusobacteria & riboflavin & Others & Riboflavin metabolism & -0.432 & $6.270 \mathrm{E}-05$ \\
\hline Leptotrichia & Fusobacteria & Urate radical & Nucleotides & Nucleotide metabolism & 0.296 & 7.684E-03 \\
\hline Leptotrichia & Fusobacteria & Vanillylmandelic acid & Benzene derivatives & Tyrosine metabolism & 0.463 & $1.520 \mathrm{E}-05$ \\
\hline Leuconostoc & Firmicutes & 5-hydroxyindoleacetic acid & Indoles & Tryptophan metabolism & -0.263 & $1.846 \mathrm{E}-02$ \\
\hline Leuconostoc & Firmicutes & Orotidine & Nucleotides & Pyrimidine metabolism & -0.360 & $1.054 \mathrm{E}-03$ \\
\hline Methyloversatilis & Proteobacteria & 2-Aminophenol & Benzene derivatives & Tryptophan metabolism & 0.229 & 4.089E-02 \\
\hline Methyloversatilis & Proteobacteria & Isocitric acid & Organic acids & Citrate cycle (TCA cycle) & 0.235 & $3.628 \mathrm{E}-02$ \\
\hline Methyloversatilis & Proteobacteria & Urate radical & Nucleotides & Nucleotide metabolism & 0.273 & $1.444 \mathrm{E}-02$ \\
\hline Mobiluncus & Actinobacteria & riboflavin & Others & Riboflavin metabolism & -0.255 & $2.243 \mathrm{E}-02$ \\
\hline Mycoplasma & Tenericutes & 2-Aminophenol & Benzene derivatives & Tryptophan metabolism & 0.229 & $4.085 \mathrm{E}-02$ \\
\hline Mycoplasma & Tenericutes & 5-hydroxyindoleacetic acid & Indoles & Tryptophan metabolism & 0.345 & $1.738 \mathrm{E}-03$ \\
\hline Mycoplasma & Tenericutes & Isocitric acid & Organic acids & Citrate cycle (TCA cycle) & 0.251 & $2.474 \mathrm{E}-02$ \\
\hline Mycoplasma & Tenericutes & Orotidine & Nucleotides & Pyrimidine metabolism & 0.274 & $1.397 \mathrm{E}-02$ \\
\hline Mycoplasma & Tenericutes & phenylacetylglycine & Amino acids & Phenylalanine metabolism & 0.439 & $4.650 \mathrm{E}-05$ \\
\hline Mycoplasma & Tenericutes & Succinic acid & Organic acids & Citrate cycle (TCA cycle) & -0.226 & 4.406E-02 \\
\hline Mycoplasma & Tenericutes & Urate radical & Nucleotides & Nucleotide metabolism & 0.284 & $1.057 \mathrm{E}-02$ \\
\hline Mycoplasma & Tenericutes & Vanillylmandelic acid & Benzene derivatives & Tyrosine metabolism & 0.402 & $2.165 \mathrm{E}-04$ \\
\hline Oribacterium & Firmicutes & Isocitric acid & Organic acids & Citrate cycle (TCA cycle) & 0.350 & $1.452 \mathrm{E}-03$ \\
\hline Paenibacillus & Firmicutes & Dopamine 3-O-sulfate & Benzene derivatives & Tyrosine metabolism & -0.248 & $2.639 \mathrm{E}-02$ \\
\hline
\end{tabular}




\begin{tabular}{|c|c|c|c|c|c|c|}
\hline Paenibacillus & Firmicutes & Isocitric acid & Organic acids & Citrate cycle (TCA cycle) & 0.367 & $8.213 \mathrm{E}-04$ \\
\hline Paenibacillus & Firmicutes & Urate radical & Nucleotides & Nucleotide metabolism & 0.330 & $2.794 \mathrm{E}-03$ \\
\hline Paenibacillus & Firmicutes & Uric acid & Nucleotides & Purine metabolism & 0.310 & $5.141 \mathrm{E}-03$ \\
\hline Paenibacillus & Firmicutes & Vanillylmandelic acid & Benzene derivatives & Tyrosine metabolism & 0.280 & $1.179 \mathrm{E}-02$ \\
\hline Parvimonas & Firmicutes & Isocitric acid & Organic acids & Citrate cycle (TCA cycle) & 0.407 & $1.777 \mathrm{E}-04$ \\
\hline Parvimonas & Firmicutes & riboflavin & Others & Riboflavin metabolism & -0.303 & $6.311 \mathrm{E}-03$ \\
\hline Peptoclostridium & Firmicutes & Acetoacetic acid & Organic acids & $\begin{array}{l}\text { Synthesis and degradation of ketone } \\
\text { bodies }\end{array}$ & 0.244 & $2.933 \mathrm{E}-02$ \\
\hline Peptoclostridium & Firmicutes & Itaconic acid & Fatty acids & C5-Branched dibasic acid metabolism & 0.265 & $1.750 \mathrm{E}-02$ \\
\hline Peptoclostridium & Firmicutes & L-2-hydroxyglutaric acid & Organic acids & Lysine degradation & 0.268 & $1.635 \mathrm{E}-02$ \\
\hline Peptoclostridium & Firmicutes & phenylacetylglycine & Amino acids & Phenylalanine metabolism & -0.243 & 2.992E-02 \\
\hline Porphyromonas & Bacteroidetes & Acetoacetic acid & Organic acids & $\begin{array}{l}\text { Synthesis and degradation of ketone } \\
\text { bodies }\end{array}$ & 0.312 & $4.906 \mathrm{E}-03$ \\
\hline Porphyromonas & Bacteroidetes & Itaconic acid & Fatty acids & C5-Branched dibasic acid metabolism & 0.308 & $5.368 \mathrm{E}-03$ \\
\hline Porphyromonas & Bacteroidetes & L-2-hydroxyglutaric acid & Organic acids & Lysine degradation & 0.309 & $5.237 \mathrm{E}-03$ \\
\hline Prevotella 7 & Bacteroidetes & 5-hydroxyindoleacetic acid & Indoles & Tryptophan metabolism & -0.272 & $1.464 \mathrm{E}-02$ \\
\hline Prevotella 7 & Bacteroidetes & Acetoacetic acid & Organic acids & $\begin{array}{l}\text { Synthesis and degradation of ketone } \\
\text { bodies }\end{array}$ & 0.308 & $5.381 \mathrm{E}-03$ \\
\hline Prevotella 7 & Bacteroidetes & Itaconic acid & Fatty acids & C5-Branched dibasic acid metabolism & 0.322 & $3.558 \mathrm{E}-03$ \\
\hline Prevotella 7 & Bacteroidetes & L-2-hydroxyglutaric acid & Organic acids & Lysine degradation & 0.332 & $2.620 \mathrm{E}-03$ \\
\hline Prevotella 7 & Bacteroidetes & L-Dopa & Amino acids & Tyrosine metabolism & -0.280 & $1.174 \mathrm{E}-02$ \\
\hline Prevotella 7 & Bacteroidetes & Orotidine & Nucleotides & Pyrimidine metabolism & -0.288 & $9.508 \mathrm{E}-03$ \\
\hline $\begin{array}{l}\text { Prevotellaceae UCG- } \\
004\end{array}$ & Bacteroidetes & $\begin{array}{l}\text { 5- } \\
\text { Hydroxyindoleacetaldehyde }\end{array}$ & Indoles & Tryptophan metabolism & -0.244 & $2.904 \mathrm{E}-02$ \\
\hline Pseudomonas & Proteobacteria & Glutamine & Amino acids & Pyrimidine metabolism & 0.230 & $3.994 \mathrm{E}-02$ \\
\hline Pseudomonas & Proteobacteria & Isocitric acid & Organic acids & Citrate cycle (TCA cycle) & 0.367 & $8.062 \mathrm{E}-04$ \\
\hline
\end{tabular}




\begin{tabular}{|c|c|c|c|c|c|c|}
\hline Pseudomonas & Proteobacteria & Normetanephrine & Benzene derivatives & Tyrosine metabolism & -0.242 & $3.042 \mathrm{E}-02$ \\
\hline Pseudomonas & Proteobacteria & riboflavin & Others & Riboflavin metabolism & -0.433 & $5.980 \mathrm{E}-05$ \\
\hline Rothia & Actinobacteria & Glutamine & Amino acids & Pyrimidine metabolism & -0.222 & 4.812E-02 \\
\hline Rothia & Actinobacteria & Hydroquinone & Benzene derivatives & Tyrosine metabolism & 0.238 & $3.333 \mathrm{E}-02$ \\
\hline Selenomonas 4 & Firmicutes & Acetoacetic acid & Organic acids & $\begin{array}{l}\text { Synthesis and degradation of ketone } \\
\text { bodies }\end{array}$ & 0.274 & $1.378 \mathrm{E}-02$ \\
\hline Selenomonas 4 & Firmicutes & Hydroquinone & Benzene derivatives & Tyrosine metabolism & 0.302 & $6.516 \mathrm{E}-03$ \\
\hline Selenomonas 4 & Firmicutes & Isocitric acid & Organic acids & Citrate cycle (TCA cycle) & 0.279 & $1.232 \mathrm{E}-02$ \\
\hline Selenomonas 4 & Firmicutes & Itaconic acid & Fatty acids & C5-Branched dibasic acid metabolism & 0.275 & $1.371 \mathrm{E}-02$ \\
\hline Selenomonas 4 & Firmicutes & L-2-hydroxyglutaric acid & Organic acids & Lysine degradation & 0.265 & $1.765 \mathrm{E}-02$ \\
\hline Selenomonas 4 & Firmicutes & riboflavin & Others & Riboflavin metabolism & -0.386 & 4.114E-04 \\
\hline Selenomonas 4 & Firmicutes & Uric acid & Nucleotides & Purine metabolism & 0.227 & $4.273 \mathrm{E}-02$ \\
\hline Silanimonas & Proteobacteria & 5-hydroxyindoleacetic acid & Indoles & Tryptophan metabolism & 0.262 & $1.866 \mathrm{E}-02$ \\
\hline Silanimonas & Proteobacteria & Isocitric acid & Organic acids & Citrate cycle (TCA cycle) & 0.265 & $1.736 \mathrm{E}-02$ \\
\hline Silanimonas & Proteobacteria & Orotidine & Nucleotides & Pyrimidine metabolism & 0.306 & $5.726 \mathrm{E}-03$ \\
\hline Solobacterium & Firmicutes & Succinic acid & Organic acids & Citrate cycle (TCA cycle) & -0.231 & $3.966 \mathrm{E}-02$ \\
\hline Staphylococcus & Firmicutes & $\begin{array}{l}\text { 5- } \\
\text { Hydroxyindoleacetaldehyde }\end{array}$ & Indoles & Tryptophan metabolism & -0.291 & 8.903E-03 \\
\hline Stomatobaculum & Firmicutes & Hydroquinone & Benzene derivatives & Tyrosine metabolism & 0.256 & $2.183 \mathrm{E}-02$ \\
\hline Treponema 2 & Spirochaetes & Acetoacetic acid & Organic acids & $\begin{array}{l}\text { Synthesis and degradation of ketone } \\
\text { bodies }\end{array}$ & 0.308 & $5.458 \mathrm{E}-03$ \\
\hline Treponema 2 & Spirochaetes & Glycine & Amino acids & Purine metabolism & 0.271 & $1.487 \mathrm{E}-02$ \\
\hline Treponema 2 & Spirochaetes & Isocitric acid & Organic acids & Citrate cycle (TCA cycle) & 0.245 & $2.827 \mathrm{E}-02$ \\
\hline Treponema 2 & Spirochaetes & Itaconic acid & Fatty acids & C5-Branched dibasic acid metabolism & 0.331 & $2.728 \mathrm{E}-03$ \\
\hline Treponema 2 & Spirochaetes & L-2-hydroxyglutaric acid & Organic acids & Lysine degradation & 0.331 & $2.685 \mathrm{E}-03$ \\
\hline
\end{tabular}




\begin{tabular}{|c|c|c|c|c|c|c|}
\hline unidentified & & & & & & \\
\hline $\begin{array}{l}\text { Clostridiales } \\
\text { vadinBB60 group }\end{array}$ & Firmicutes & Isocitric acid & Organic acids & Citrate cycle (TCA cycle) & 0.333 & $2.506 \mathrm{E}-03$ \\
\hline unidentified & & & & & & \\
\hline Clostridiales & Firmicutes & riboflavin & Others & Riboflavin metabolism & -0.271 & $1.494 \mathrm{E}-02$ \\
\hline vadinBB60 group & & & & & & \\
\hline $\begin{array}{l}\text { unidentified Family } \\
\text { XIII }\end{array}$ & Firmicutes & Hydroquinone & Benzene derivatives & Tyrosine metabolism & 0.249 & $2.566 \mathrm{E}-02$ \\
\hline $\begin{array}{l}\text { unidentified Family } \\
\text { XIII }\end{array}$ & Firmicutes & Isocitric acid & Organic acids & Citrate cycle (TCA cycle) & 0.419 & $1.117 \mathrm{E}-04$ \\
\hline $\begin{array}{l}\text { unidentified Family } \\
\text { XIII }\end{array}$ & Firmicutes & riboflavin & Others & Riboflavin metabolism & -0.298 & 7.191E-03 \\
\hline $\begin{array}{l}\text { unidentified Family } \\
\text { XIII }\end{array}$ & Firmicutes & Urate radical & Nucleotides & Nucleotide metabolism & 0.244 & 2.887E-02 \\
\hline $\begin{array}{l}\text { unidentified } \\
\text { Porphyromonadaceae }\end{array}$ & Bacteroidetes & Isocitric acid & Organic acids & Citrate cycle (TCA cycle) & 0.376 & $5.828 \mathrm{E}-04$ \\
\hline $\begin{array}{l}\text { unidentified } \\
\text { Porphyromonadaceae }\end{array}$ & Bacteroidetes & riboflavin & Others & Riboflavin metabolism & -0.236 & $3.507 \mathrm{E}-02$ \\
\hline $\begin{array}{l}\text { unidentified } \\
\text { Porphyromonadaceae }\end{array}$ & Bacteroidetes & Succinic acid & Organic acids & Citrate cycle (TCA cycle) & -0.240 & $3.176 \mathrm{E}-02$ \\
\hline Veillonella & Firmicutes & Isocitric acid & Organic acids & Citrate cycle (TCA cycle) & 0.355 & $1.234 \mathrm{E}-03$ \\
\hline Veillonella & Firmicutes & Itaconic acid & Fatty acids & C5-Branched dibasic acid metabolism & 0.222 & 4.739E-02 \\
\hline Veillonella & Firmicutes & L-2-hydroxyglutaric acid & Organic acids & Lysine degradation & 0.221 & 4.850E-02 \\
\hline Veillonella & Firmicutes & riboflavin & Others & Riboflavin metabolism & -0.335 & 2.359E-03 \\
\hline
\end{tabular}

